RI-2-96

TAUP 2175-94

IASSNS-96/31

February 7, 2008

\title{
Generalization of the Coleman-Mandula Theorem to Higher Dimension
}

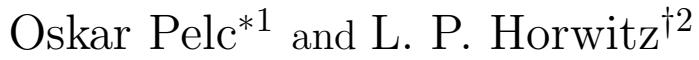 \\ * Racah Institute of Physics, The Hebrew University \\ Jerusalem, 91904, Israel \\ ${ }^{\dagger}$ School of Natural Sciences, Institute for Advanced Study, \\ Princeton, N.J. 08540, USA
}

\begin{abstract}
The Coleman-Mandula theorem, which states that space-time and internal symmetries cannot be combined in any but a trivial way, is generalized to an arbitrarily higher spacelike dimension. Prospects for further generalizations of the theorem (space-like representations, larger time-like dimension, infinite number of particle types) are also discussed. The original proof relied heavily on the Dirac formalism, which was not well defined mathematically at that time. The proof given here is based on the rigorous version of the Dirac formalism, based on the theory of distributions. This work serves also to demonstrate the suitability of this formalism for practical applications.
\end{abstract}

PACS codes: 3.65.Pm, 3.65.Db, 11.10.Cd, 11.10.Kk, 11.30.Cp, 11.80.-m

\footnotetext{
${ }^{1}$ E-mail: oskar@shum.cc.huji.ac.il

${ }^{2}$ E-mail: horwitz@sns.ias.edu

On sabbatical leave from the School of Physics and Astronomy, Raymond and Beverly Sackler Faculty of Exact Sciences, Tel-Aviv University, Ramat-Aviv, Israel; also at Department of Physics, Bar-Ilan University, Ramat Gan, Israel.
} 


\section{Introduction}

\section{I.1 The Coleman Mandula theorem}

Symmetry plays a key role in modern physics, and in the investigation of the foundations of physics in particular. Symmetry considerations were found extremely useful in the understanding of physical phenomena (e.g. particle classification, selection rules) and in the formulation of theories describing a given physical system. The choice of a symmetry group of the system determines to a great extent its properties.

In a relativistic theory, this group must contain (as a subgroup) the Poincaré group: translations, rotations and Lorentz transformations. In 1967, Coleman and Mandula [1] proved a theorem which puts a severe restriction on the groups that can serve as physical symmetry groups.

They proved that (this is a loose statement of the theorem; a more precise one will follow): if

1. the $\mathrm{S}$ matrix is not trivial and is such that the elastic scattering amplitudes are analytic functions of $s$ (the squared center-of-mass energy) and $t$ (the squared momentum transfer),

2. the mass spectrum of the one particle states is a (possibly infinite) set of isolated points, all positive, and there is a finite number of particle types with a given mass,

3. $\mathrm{G}$ is a connected symmetry group of the $\mathrm{S}$ matrix which contains the Poincaré group and is generated, at least locally, by generators ("infinitesimal symmetry transformations") representable as integral operators in "momentum space" with distributions as kernels,

then

$\mathrm{G}$ is locally isomorphic to the direct product of the Poincaré group and an internal symmetry group ("internal" means symmetries that commute with the Poincaré group).

The implications of the theorem are far reaching. It implies that, at least in the domain of classical groups, and under the stated assumptions of the theorem, there is no connection between the space-time symmetries and the other symmetries - those "mixing" different particle "types" (e.g. charge, flavour, color). This means that the properties and structure of the Poincaré group are of no help in choosing the set of other symmetries. But the most important implication is that symmetries cannot relate particles with different mass and spin and thus the hope to describe the full variety of particle types through symmetry considerations was destroyed.

The possibility of supersymmetry was not envisaged by Coleman and Mandula and the introduction of supersymmetries in 1973 (by Volkov and Akulov [2] and independently 
by Wess and Zumino [3]) offered a bypass of their theorem. However, most of the ideas in the theorem apply to supersymmetries as well and in 1974 they were exploited by Haag, Łopuszański and Sohnius [4] to put severe restrictions on the possible supersymmetries.

The set of supersymmetries constitutes a "Graded Lie Algebra" (GLA) which is a generalization of a Lie algebra and thus the supersymmetries are interpreted as infinitesimal symmetry transformations. A GLA $\mathcal{A}$ is a direct sum of two vector spaces: The space $\mathcal{A}_{0}$ of even ("bosonic") elements, related by commutators, and the space $\mathcal{A}_{1}$ of odd ("fermionic") elements, related by anticommutators. ( $\mathcal{A}_{0}$ is an ordinary Lie algebra so the Coleman-Mandula theorem applies to it directly)

Haag et al. proved that (this is also a loose statement): if

1. assumptions 1 and 2 of the Coleman-Mandula theorem are satisfied,

2. the elements of $\mathcal{A}$ are (infinitesimal) symmetries of the S-matrix, then

1. the bosonic generators, except those of the Poincaré group are all translation invariant Lorentz scalars (i.e., generators of internal symmetries),

2. the fermionic generators are translation invariant Majorana spinors,

3. the commutators and anticommutators are determined to a large extent (details will not be given here); in particular, if there are no internal symmetries, they are determined uniquely.

\section{I.2 Extension of the Theorem}

We discuss in the following some motivations for extending the Coleman-Mandula theorem to higher dimensions.

Manifestly covariant relativistic quantum mechanics has been formulated as a theory of the evolution of events in space-time [5, 6]. The wave functions are square integrable functions on the four dimensional space-time. The principle strategy in developing the theory is to take the Schrödinger formulation of non-relativistic quantum mechanics in three dimensional space and generalize it, with appropriate interpretations, to the relativistic space-time of events. In particular, in [5], the wave functions are parametrized by a universal world time $\tau$ which is the analogue of the non-relativistic time as an evolution parameter, and evolution in $\tau$ is generated by a Hamiltonian-like operator $K$, which is a function of space and time coordinates and their canonical conjugate coordinates - momentum and energy. (This must be a Lorentz-invariant function, in order to have the manifest relativistic covariance of dynamical evolution.) For example, for a spinless particle, one may take

$$
K=\frac{p_{\mu} p^{\mu}}{2 M}+V(\rho)
$$

(with the signature -+++ ) where $V(\rho)$ is a real function of $\rho \equiv x_{\mu} x^{\mu}=x^{2}-t^{2}$ and M is a parameter (with the dimension of mass). 
It is important to notice that here $\left\{p_{\mu}\right\}$ are four independent variables, so the mass $m \equiv \sqrt{p_{\mu} p^{\mu}}$ is a dynamical variable and not a fixed parameter. It is obviously connected with the fact that space and time coordinates are all independent dynamical variables [5], so it is an intrinsic property of every theory of this kind [6].

Recalling the Coleman-Mandula theorem, we see that it is not valid here because it assumes a discrete mass spectrum. Indeed, in the formulation of a theory of electromagnetic interactions [5], one gets, through the requirement of gauge invariance, (in the same way that classical electromagnetism introduced the Lorentz symmetry), a 5-D symmetry group: $\mathcal{P}(4,1)$ or $\mathcal{P}(3,2)$ (where $\mathcal{P}(r, s)$ denotes the inhomogeneous pseudo orthogonal group of signature $(r, s)$ ) and this is not a direct product of the Poincaré group $\mathcal{P}(3,1)$ and another group [7]. So the Coleman-Mandula theorem and its extension to supersymmetry are not available here to guide us in the choice of the symmetries of a system [8].

However, a further analysis suggests that Coleman-Mandula's assumptions can be generalized to this framework in accordance with all the other changes. The assumptions about the mass spectrum were actually assumptions about the number of particle types, since a particle type was identified with an irreducible representation of the Poincaré group in the Hilbert space of one-particle states and such a representation has a definite mass. But in the higher dimensional theory, a change in mass is not interpreted as a change of particle type. For example, in the free $(V=0)$ spinless theory, $m \equiv \sqrt{p_{\mu} p^{\mu}}$ is a constant of the motion and with an appropriate calibration it can be put equal to $M$. It is therefore possible to interpret $M$ (or some function of $M$ ) as the intrinsic "mass" of the particle described, while interactions take the particle off "mass shell".

Thus, a particle type cannot be identified here with an irreducible representation of $\mathcal{P}(3,1)$. But if we have a higher-dimensional symmetry group, as suggested by the generalized form of electromagnetism, an irreducible representation of this group may be an adequate candidate to be identified with a particle type. So it seems that in the theorem that should be the analogue of the Coleman-Mandula theorem here, the Poincaré group should be replaced by the larger symmetry group and the mass $m \equiv \sqrt{p_{\mu} p^{\mu}}$ should be replaced by the analogue Casimir operator in the larger group. This is the type of theorem that will be proved in this work. In this theorem, the $\mathcal{P}(3,1)$ group will be replaced by $\mathcal{P}(r, 1)$. A further generalization to $\mathcal{P}(r, s)$ would also be desireble, however in the consideration of more than one time-like direction, there are several complications and it is not clear if such a generalization is possible. This issue is discussed in subsection 4.1 .

This work may also be relevant to the recent developments of Kaluza-Klein type theories for the construction of grand unified or string theories, where one deals with generalized space-time manifold of many dimensions (with signature e.g. $(d-1,1)$ ). However, in applying the theorem proved here to these cases, the meaning of the assumptions on the spectrum of the Casimir operator introduced above must be carefully considered.

There exist generalizations of the Coleman-Mandula theorem in other directions: candidates for symmetry generators defined by conserved currents are studied in [9]; more general generators and non-local charges are considered in [10]; Galilean field theories are studied in [11] and massless particles are considered in [12]. 


\section{I.3 The Mathematical Formulation}

The proof of the original Coleman-Mandula theorem relies heavily on the "bra" and "ket" formalism of Dirac, using a basis of "plane-wave states", matrix elements of operators in this basis, $\delta$-functions etc. . The original formalism, due to Dirac [13] (confined to a Hilbert space) is not well defined and thus is not suitable for a formal mathematical proof. Here we use a rigorous version of this formalizm [14], based on replacing the traditional Hilbert space by a Gel'fand triple. In [15] this approach was used to construct the basis of planewave states in a (well-defined) generalized sense and to give a precise meaning to other concepts used in this context, such as generators of symmetry and scattering amplitudes. The results of [15] are extensively used throughout this work and they are summarized in appendix A.

\section{I.4 The Structure of This Work}

We begin, in section 2, with a concise description of the main concepts needed for the formulation and proof of the theorem - the space of states, the S-matrix, symmetries etc. - Then a precise statement of the theorem is given. In section 3 the theorem is proved along the lines described in [回]. In section 4 we comment on the theorem proved and on possible further extensions. Appendix A collects the notation and definition of all the concepts used in the text and in appendix B some technical properties of two-body scattering are derived.

\section{The Theorem}

\section{II.1 The Scenario}

In relativistic scattering theory, the space $\mathcal{H}_{s}$ of physical states is a direct sum of (complex, separable) Hilbert spaces

$$
\mathcal{H}_{s}=\bigoplus_{n=0}^{\infty} \mathcal{H}_{s}^{(n)}
$$

where $\mathcal{H}_{s}^{(n)}$ is the space of $n$-particle states (thus called " $n$-particle space") and is (isomorphic to) a closed subspace of the completed tensor product of $n$ one-particle spaces:

$$
\mathcal{H}_{s}^{(n)} \subset \mathcal{H}^{(n)}=\overline{\bigotimes_{1}^{n} \mathcal{H}^{(1)}} .
$$

The elements of $\mathcal{H}_{s}^{(n)}$ are those elements of $\mathcal{H}^{(n)}$ which have the right symmetry properties with respect to the exchange of identical particles. This symmetry is not relevant in the subsequent analysis, therefore we consider the larger space

$$
\mathcal{H}=\bigoplus_{n=0}^{\infty} \mathcal{H}^{(n)}
$$


According to the rigorized Dirac formalism [14], $\mathcal{H}$ belongs to a Gel'fand triple $\left(\Phi, \mathcal{H}, \Phi^{\prime}\right)$ of topological vector spaces, where $\Phi$ is embedded in $\mathcal{H}$ continuously as a dense subspace and $\Phi^{\prime}$ is the (strong) dual of $\Phi$. This also induces a corresponding Gel'fand triple for any closed subspace of $\mathcal{H}$

The S-matrix $S$ is assumed to be a unitary operator on $\mathcal{H}_{s}$. It can be identified as an element of $L^{\times}\left(\Phi ; \Phi^{\prime}\right)$ - a continuous antilinear map from $\Phi$ to $\Phi^{\prime}$ - and as such, it has a corresponding kernel $\langle S\rangle$ (more precisely - kernels $\left\langle S^{(m, n)}\right\rangle \in \mathcal{D}\left(\Omega^{m} \times \Omega^{n}\right)$ ).

A symmetry transformation of the S-matrix is defined to be a unitary or antiunitary operator $U$ in $\mathcal{H}_{s}$ which satisfies:

1. $\mathcal{H}^{(1)}$ is $U$-invariant, i.e. $U$ turns one-particle states into one-particle states.

2. $U$ acts on many-particle states in accordance to their relation to the tensor product of one-particle states:

$$
U\left(f_{1} \otimes \cdots \otimes f_{n}\right)=\left(U f_{1}\right) \otimes \cdots \otimes\left(U f_{n}\right)
$$

(and thus, according to property $1, \forall n, \mathcal{H}_{s}^{(n)}$ is $U$-invariant).

3. $U$ commutes with $S$.

Under the assumptions of the theorem, including translation invariance of $S$, it can be shown that its kernel $\langle S>$ has the following form

$$
<S^{(m, n)}>=<I^{(m, n)}>-i(2 \pi)^{d} \delta^{d}\left(\sum_{1}^{m} q_{j}-\sum_{1}^{n} p_{i}\right)<T^{(m, n)}>
$$

where $d$ is the dimension of the momentum space, $-i(2 \pi)^{d}$ is a conventional normalization factor and $\langle T\rangle$ is a generalized function of the momenta $q_{j}, p_{i}$, restricted to the domain defined by the constraint

$$
\sum_{1}^{m} q_{j}-\sum_{1}^{n} p_{i}=0
$$

The values of $\langle T\rangle$ are called "scattering amplitudes".

\section{II.2 The Statement of the Theorem}

\section{The assumptions:}

(See remarks in section 4 )

1. $G$ is a connected group of symmetries of the S-matrix $S$.

2. (Lorentz invariance) $G$ contains a subgroup $\mathcal{P}_{0}^{\prime}$, locally isomorphic to $\mathcal{P}(r, 1)$, the inhomogeneous pseudo orthogonal group of Lorentzian signature $(r, 1)$, where $r \geq 3$. 
3. All particle types correspond to positive-energy time-like representations of $\mathcal{P}$, the universal covering group of $\mathcal{P}_{0}^{\prime}$ (i.e. the spectrum $\hat{\mathcal{T}}_{F}$ of the momentum operator $P$ in the space $\mathcal{H}^{(1)}$ of one-particle states is contained in the forward light cone:

$$
\left.\forall p \in \hat{\mathcal{T}}_{F} \quad, \quad p_{\mu} p^{\mu}>0 \quad, \quad E \equiv p^{0}>0\right) .
$$

4. (Particle finiteness) The number of particle types is finite.

5. (Existence of generators) $G$ is generated, at least locally, by generators represented in the one-particle space $\mathcal{H}^{(1)}$ by (generalized) integral operators in momentum space, with distributions as kernels (i.e. there is a neighborhood of the identity in $G$, such that each element in that neighborhood belongs to a one parameter subgroup $g(t)$ and there exists $A_{g} \in L^{\times}\left(\Phi^{(1)} ; \Phi^{(1)^{\prime}}\right)$ that satisfies:

$$
\left(\psi\left|A_{g} \varphi>=\frac{1}{i} \frac{d}{d t}\left(\psi, U^{(1)}(g(t)) \varphi\right)\right|_{t=0} \quad, \quad \forall \varphi, \psi \in \Phi^{(1)}\right.
$$

where $U^{(1)}$ is the representation of $G$ in $\left.\mathcal{H}^{(1)}\right)$.

6. (Analyticity) The scattering amplitudes $\langle T\rangle$ are regular functions of the momenta. The amplitudes $<T^{(2,2)}>$ for scattering between two-particle states

$$
\left(p_{1}, p_{2}\right) \longrightarrow\left(p_{1}^{\prime}, p_{2}^{\prime}\right)
$$

are analytic functions of (see appendix B.1)

$$
s=\left(p_{1}+p_{2}\right)^{2} \text { and } t=\left(p_{1}^{\prime}-p_{1}\right)^{2}
$$

in some neighborhood of the physical region.

7. (The occurrence of elastic scattering) The amplitudes for elastic scattering of two particles do not vanish identically.

\section{The result obtained:}

$G$ is locally isomorphic to the direct product of $\mathcal{P}_{0}^{\prime}$ and an internal symmetry group.

\section{The Proof}

The proof of the theorem is divided naturally to three parts. Each part is built as a sequence of lemmas and ends with a proposition which states the final results of that part. The first two parts are completely independent and the last part uses only the propositions of the preceding parts. 
The proof is based on an analysis of the generators of $G$, concluding that these are always sums of generators of $\mathcal{P}$ and generators of internal symmetry transformations. The analysis will be performed on a space $\mathcal{A}$ of operators that includes the set of generators as a subspace (but a priori may be larger). In appendix B.2 it is shown that, $\Phi^{(2)}$ is $S^{(2,2)}$-invariant. This allows the following definition:

Definition: $\mathcal{A}$ is the set $\{A\}$ of elements of $L^{\times}\left(\Phi^{(1)} ; \Phi^{(1)^{\prime}}\right)$ for which $A^{\dagger}$ "commutes" with the $S$-matrix $S$ in $\Phi^{(1)} \times \Phi^{(1)}$ in the sense:

$$
\left(S^{*} \psi\left|A^{\dagger} \varphi>=<A \psi\right| S \varphi\right), \forall \varphi, \psi \in \Phi^{(1)} \times \Phi^{(1)}
$$

where

$$
A \varphi:=(A \otimes I+I \otimes A) \varphi=\left(A \varphi_{1}\right) \otimes \varphi_{2}+\varphi_{1} \otimes\left(A \varphi_{2}\right)
$$

The properties of $\mathcal{A}$ :

1. Each generator of $G$ is a (self adjoint) element of $\mathcal{A}$ (see appendix A.8).

2. $\mathcal{A}$ is a vector space and it is a closed subspace of $\mathcal{D}^{\prime}(\Omega \times \Omega)$ : each sum or integral of elements of $\mathcal{A}$ which converges in $\mathcal{D}^{\prime}(\Omega \times \Omega)$ is in $\mathcal{A}$.

3. If $A \in \mathcal{A}$ and $(\Lambda, a) \in \mathcal{P}$ then $U^{(1)}(\Lambda, a)^{\prime} A U^{(1)}(\Lambda, a) \in \mathcal{A}$ (since $\Phi^{(1)}$ is $U(\mathcal{P})$ invariant and $S$ commutes with $U(\mathcal{P}))$.

4. If $A, B \in \mathcal{A}, \Phi^{(1)}$ is invariant under $A$ and $B$ and $A B, B A \in L^{\times}\left(\Phi^{(1)} ; \Phi^{(1)^{\prime}}\right)$ then $[A, B] \in \mathcal{A}$. (Notice that $\mathcal{A}$ is not necessarily a Lie algebra: for $A, B \in \mathcal{A}, A B$ or $B A$ may be undefined.)

\section{Part 1}

This part analyzes the dependence of elements of $\mathcal{A}$ on the momentum. Let $A$ be an element of $\mathcal{A}$ and $f$ a test function on the momentum space (an element of $\mathcal{D}(\hat{\mathcal{T}}))$. We define

$$
\left.f \cdot A:=\int_{\mathcal{T}} d a U(1, a)^{\prime} A U(1, a)\right) \tilde{f}(a)
$$

where $\tilde{f}$ is the Fourier transform of $f$ :

$$
\tilde{f}(a):=\int_{\hat{\mathcal{I}}} \frac{d p}{(2 \pi)^{d}} f(p) e^{i p \cdot a} .
$$

\section{Lemma 1:}

$f \cdot A$ is in $\mathcal{A}$ and its matrix elements are:

$$
<p^{\prime}|f \cdot A| p>=f\left(p^{\prime}-p\right)<p^{\prime}|A| p>
$$

which implies that

$$
\operatorname{supp}[(f \cdot A) \varphi] \subset \operatorname{supp}(\varphi)+\operatorname{supp}(f) .
$$


Proof:

For each $\varphi, \psi \in \Phi^{(1)}$

$$
\begin{aligned}
(\psi \mid(f \cdot A) \varphi>= & \\
= & \int_{\mathcal{T}} d a \tilde{f}(a)\left(\psi \mid U(1, a)^{\prime} A U(1, a) \varphi>=\right. \\
= & \int_{\mathcal{T}} d a \int_{\hat{\mathcal{T}}} \frac{d q}{(2 \pi)^{d}} f(q) e^{i q \cdot a} \\
& \quad \int d \mu\left(p^{\prime}\right) d \mu(p) e^{-i p^{\prime} \cdot a}\left(\psi\left|p^{\prime}><p^{\prime}\right| A|p><p| \varphi\right) e^{i p \cdot a} \\
= & \int d \mu\left(p^{\prime}\right) d \mu(p)\left(\psi\left|p^{\prime}><p^{\prime}\right| A|p><p| \varphi\right) \\
& \quad \int_{\mathcal{T}} d a \int_{\hat{\mathcal{T}}} \frac{d q}{(2 \pi)^{d}} f(q) e^{i\left[q-\left(p^{\prime}-p\right)\right] \cdot a} \\
= & \int d \mu\left(p^{\prime}\right) d \mu(p)\left(\psi\left|p^{\prime}><p^{\prime}\right| A|p><p| \varphi\right) f\left(p^{\prime}-p\right)
\end{aligned}
$$

where the second to last equality is due to Fubini's theorem for distributions. The last stage identifies $\langle f \cdot A>$ as a distribution on $\Omega \times \Omega$ (an element of $\mathcal{D}^{\prime}(\Omega \times \Omega)$ ), thus $f \cdot A$ is in $\mathcal{A}$ (according to properties 2 and 3 of $\mathcal{A}$ ).

Now if $p^{\prime} \in \operatorname{supp}[(f \cdot A) \varphi]$ then there exists $p \in \operatorname{supp}(\varphi)$ for which $<p^{\prime}|f \cdot A| p>\neq 0$. Using ([I.5), this implies that $f\left(p^{\prime}-p\right) \neq 0$, which means that $\Delta p \equiv p^{\prime}-p$ is in $\operatorname{supp}(f)$, so for each $p^{\prime} \in \operatorname{supp}[(f \cdot A) \varphi]$ we have

$$
p^{\prime}=p+\Delta p, \text { where } p \in \operatorname{supp}(\varphi) \text { and } \Delta p \in \operatorname{supp}(f) \text {. }
$$

Let

$0 \neq \Delta p_{0} \in \hat{\mathcal{T}}, \rho>0, \Delta R$ a ball of radius $\rho$ around $\Delta p_{0}$ and $f$ a test function on $\hat{\mathcal{T}}$, with support contained in $\Delta R$.

It will be shown that in such a situation, for $\rho$ sufficiently small, $f \cdot A$ vanishes because $f \cdot A \neq 0$ would contradict the occurrence of elastic scattering (assumption 7 of the theorem). To show this, we will construct regions in $\hat{\mathcal{T}}$ such that states with support in these regions are not connected by the S-matrix. For this it is needed to analyze the action of $f \cdot A$ in momentum space. The momentum support of elements of $\Phi^{(1)}$ (and thus also of elements of $\Phi^{(1)^{\prime}}$ ) is contained in "the physical region" $\hat{\mathcal{T}}_{F}$, which is a (finite) union of orbits $\left\{\hat{\mathcal{T}}_{m}\right\}_{m \in \mathcal{M}}$ (each orbit being an $r$ dimensional hyperboloid, see figure [II.1). According to eq. ([II.6), to have $(f \cdot A) \varphi \neq 0$ for some $\varphi \in \Phi^{(1)}$, $\operatorname{supp}(\varphi)$ must contain $p \in \hat{\mathcal{T}}_{F}$ for which there exists $\Delta p \in \Delta R$ that satisfies $p+\Delta p \in \hat{\mathcal{T}}_{F}$. In other words, $p+\Delta R$ must intersect $\hat{\mathcal{T}}_{F}$ non-trivially. The set of all points in $\hat{\mathcal{T}}_{m}$ satisfying this condition is

$$
R_{m}:=\hat{\mathcal{T}}_{m} \cap\left(\hat{\mathcal{T}}_{F}-\Delta R\right) .
$$


Figure III.1: The physical region $\hat{\mathcal{T}}_{F}$

We have therefore shown that

$$
R:=\bigcup_{m \in \mathcal{M}} R_{m}
$$

obeys:

\section{Lemma 2:}

$$
\text { If } \varphi \in \Phi^{(1)} \text { is such that } R \cap \operatorname{supp}(\varphi)=\emptyset \text { then }(f \cdot A) \varphi=0 .
$$

This lemma will be used to construct states annihilated by $f \cdot A$. To do this, we must describe $R$ :

$$
R_{m}=\hat{\mathcal{T}}_{m} \cap\left(\hat{\mathcal{T}}_{F}-\Delta p_{0}+\left(\Delta p_{0}-\Delta R\right)\right)
$$

and $\Delta p_{0}-\Delta R$ is a $\rho$-neighborhood of the origin so $\hat{\mathcal{T}}_{F}+\left(\Delta p_{0}-\Delta R\right)$ is a $\rho$-neighborhood of $\hat{\mathcal{T}}_{F}$ and thus $R_{m}$ is a neighborhood of $\hat{\mathcal{T}}_{m} \cap\left(\hat{\mathcal{T}}_{F}-\Delta p_{0}\right)$ in $\hat{\mathcal{T}}_{m}$. The situation is described in figure [II.2. This is a cross section of the momentum space at the plane $\left(p_{x}, E\right)$ (where the $x$ axis was chosen in the direction of $\Delta \vec{p}_{0}$ ). Each shaded area is (the cross section of) $\hat{\mathcal{T}}_{m_{\alpha}}-\Delta R$ (for some representative orbit $\hat{\mathcal{T}}_{m_{\alpha}}$ ) which is identified as a $\rho$-neighborhood of $\hat{\mathcal{T}}_{m_{\alpha}}-\Delta p_{0}$ (denoted by a dashed line). In this cross section, $R_{m}$ is a union of intervals, the intersection of the hyperbola $\hat{\mathcal{T}}_{m}$ with the shaded areas. Since this is a cross section, each interval represents a band in the full momentum space. These bands are described in figure [11.3. This is a projection of $\hat{\mathcal{T}}_{m}$ on the hyperplane orthogonal to the $E$-axis (since the situation is rotationally symmetric around $\Delta \vec{p}_{0}, p_{y}$ can represent all the space-like directions orthogonal to $\left.p_{x}\right)$.

Explicitly, $\hat{\mathcal{T}}_{m} \cap\left(\hat{\mathcal{T}}_{m_{\alpha}}-\Delta p\right)$ is the solution of

$$
E^{2}=m^{2}+\vec{p}^{2} ;(E+\Delta E)^{2}=m_{\alpha}^{2}+(\vec{p}+\Delta \vec{p})^{2} .
$$


Figure III.2: A cross section of the momentum space at the plane $\left(p_{x}, E\right)$

Figure III.3: A projection of $\hat{\mathcal{T}}_{m}$ on the $\vec{p}$-hyperplane 
In the $\left(p_{x}, p_{y}\right)$ plane it is characterized by the equation

$$
\begin{gathered}
\Delta E^{2} p_{y}^{2}+\Delta m^{2} p_{x}^{2}+\left(\Delta m^{2}+m^{2}-m_{\alpha}^{2}\right) \Delta p p_{x}= \\
\frac{1}{4}\left(\Delta m^{2}+m^{2}-m_{\alpha}^{2}\right)^{2}-\Delta E^{2} m^{2}
\end{gathered}
$$

(where $\Delta m^{2}:=\Delta E^{2}-\Delta p^{2}, \Delta p:=\|\Delta \vec{p}\|=\Delta p_{x}$ )

- for $\Delta m^{2}=0$ :

$$
\Delta p^{2}\left(p_{y}^{2}+m^{2}\right)+\left(m^{2}-m_{\alpha}^{2}\right)\left[\Delta p p_{x}-\frac{1}{4}\left(m^{2}-m_{\alpha}^{2}\right)\right]=0
$$

- for $\Delta m^{2} \neq 0$ :

$$
\begin{gathered}
\Delta E^{2} p_{y}^{2}+\Delta m^{2}\left(p_{x}+\frac{\Delta p}{2} \frac{\Delta m^{2}+m^{2}-m_{\alpha}^{2}}{\Delta m^{2}}\right)^{2}= \\
\frac{\Delta E^{2}}{\Delta m^{2}}\left[\frac{1}{4}\left(\Delta m^{2}+m^{2}-m_{\alpha}^{2}\right)^{2}-\Delta m^{2} m^{2}\right]
\end{gathered}
$$

In figures $\llbracket 11.2$ and $[11.3$ two situations are described:

1. $\Delta m^{2}>0$ : the intersection (when not empty) is bounded - elliptic.

2. $\Delta m^{2} \leq 0$ : the intersection (when not empty) is unbounded however it is always bounded from the direction $-\Delta E \Delta \vec{p}$ (i.e. in the direction of $\Delta \vec{p}$ if $\Delta E$ is negative and in the opposite direction if $\Delta E$ is positive).

- For $\Delta m^{2}=0$ this is a parabola.

- For $\Delta E^{2}=0$ this is a straight line parallel to the $y$ axis.

- Otherwise it is a hyperbola (only one branch).

The width of the bands depends on $\rho$ and it may become infinite for $\rho$ too large (e.g. if $\Delta R$ contains the origin, $R$ will obviously cover all of $\hat{\mathcal{T}}_{F}$ ). We choose $\rho$ small enough so that the band will be bounded from the direction $-\Delta E \Delta \vec{p}$.

In the following, $(f \cdot A)^{\dagger}$ must be considered as well as $f \cdot A$. Using eq. ([11.5) one obtains:

$$
(f \cdot A)^{\dagger}=f^{\dagger} \cdot A^{\dagger} \text { where } f^{\dagger}(p):=\overline{f(-p)}, \forall p .
$$

Let $R^{\dagger}$ and $R_{m}^{\dagger}$ denote the regions in $\hat{\mathcal{T}}_{F}$ that correspond to $(f \cdot A)^{\dagger}$ as $R$ and $R_{m}$ to $f \cdot A$.

Now it is possible to proceed with the proof. Let $p_{0}^{\prime} \in \hat{\mathcal{T}}_{m}$ for some $m \in \mathcal{M}$.

\section{Lemma 3:}

There exist three different momenta $p_{0}, q_{0}, q_{0}^{\prime} \in \hat{\mathcal{T}}_{m}$ which are not in $\bar{R} \cup \overline{R^{\dagger}}$

$\left(\bar{R}\right.$ denotes the closure of $R$ in $\hat{\mathcal{T}}_{F}$ and the same for $\left.\overline{R^{\dagger}}\right)$ and satisfy

$$
p_{0}+q_{0}=p_{0}^{\prime}+q_{0}^{\prime}
$$


Proof:

$R_{m}$ is a finite union of bands concentrated around $\hat{\mathcal{T}}_{m} \cap\left(\hat{\mathcal{T}}_{F}-\Delta p\right)$. The analysis above showed that each band is bounded from the direction $-\Delta E_{0} \Delta \vec{p}_{0}$ and thus so is $R_{m}$. To consider $R_{m}^{\dagger}$, all that is needed is to change $\Delta p_{0} \longrightarrow$ $-\Delta p_{0}$ (according to eq. (III.14) ). This leaves $-\Delta E_{0} \Delta \vec{p}_{0}$ unchanged so $R_{m}^{\dagger}$ is bounded from the same direction as $R_{m}$. Thus $\overline{R_{m}} \cup \overline{R_{m}^{\dagger}}$ is concentrated in a "half-hypersurface", and $p_{0}, q_{0}, q_{0}^{\prime}$ can always be chosen in the other half.

With $p_{0}, q_{0}$ and $q_{0}^{\prime}$ of lemma 3 , we choose neighborhoods $R_{p}, R_{q}$ of $p_{0}$ and $q_{0}$ respectively in $\hat{\mathcal{T}}_{m}$ such that $R_{p}, R_{q}, R_{q^{\prime}}:=\left(R_{p}+R_{q}-p_{0}^{\prime}\right) \cap \hat{\mathcal{T}}_{m}$ and $\overline{R_{m}} \cup \overline{R_{m}^{\dagger}}$ are disjoint. It is easy to see that such a choice is possible. Moreover, $R_{q^{\prime}}$ thus defined is a neighborhood of $q_{0}^{\prime}$ in $\hat{\mathcal{T}}_{m}$. (This is because the tangent hyperplanes to $\hat{\mathcal{T}}_{m}$ at $p_{0}$ and $q_{0}$ are not parallel so $R_{p}+R_{q}-p_{0}^{\prime}$ is a neighborhood of $q_{0}^{\prime}$ in $\hat{\mathcal{T}}$.)

Now let $\chi \in \Phi^{(1)}$ and denote $\varphi^{\prime}:=(f \cdot A) \chi\left(\in \Phi^{(1)^{\prime}}\right)$.

\section{Lemma 4:}

If $\varphi, \psi, \psi^{\prime} \in \Phi^{(1)}$ have support in $R_{p}, R_{q}$ and $R_{q^{\prime}}$ resp. then:

$$
\left.<\varphi^{\prime} \otimes \psi^{\prime} \mid(S-I)(\varphi \otimes \psi)\right)=0 \text {. }
$$

Proof:

Since $R_{q}$ and $R_{q^{\prime}}$ are disjoint, we have $\left(\psi^{\prime} \mid \psi\right)=0$ and thus

$$
\left.\left.<\varphi^{\prime} \otimes \psi^{\prime} \mid I(\varphi \otimes \psi)\right)=<\varphi^{\prime} \mid \varphi\right)\left(\psi^{\prime} \mid \psi\right)=0 .
$$

Since $R_{p}, R_{q}$ and $R_{q^{\prime}}$ don't intersect $R$ and $R^{\dagger}, \varphi, \psi$ and $\psi^{\prime}$ are annihilated by $f \cdot A$ and $(f \cdot A)^{\dagger}$ (according to lemma 2 ) so:

$$
(f \cdot A)\left(\chi \otimes \psi^{\prime}\right)=[(f \cdot A) \chi] \otimes \psi^{\prime}+\chi \otimes\left[(f \cdot A) \psi^{\prime}\right]=\varphi^{\prime} \otimes \psi^{\prime}
$$

and

$$
(f \cdot A)^{\dagger}(\varphi \otimes \psi)=0 .
$$

Combining this with the commutativity of $(f \cdot A)^{\dagger}$ with $S$ we have:

$$
\begin{aligned}
& \left.<\varphi^{\prime} \otimes \psi^{\prime} \mid S(\varphi \otimes \psi)\right)= \\
& \left.\quad=<(f \cdot A)\left(\chi \otimes \psi^{\prime}\right) \mid S(\varphi \otimes \psi)\right)= \\
& \quad=\left(S^{*}\left(\chi \otimes \psi^{\prime}\right) \mid(f \cdot A)^{\dagger}(\varphi \otimes \psi)>=0\right.
\end{aligned}
$$

\section{Lemma 5:}

$f \cdot A=0$. 
Proof:

According to lemma 4 (and using eq. A.35)

$$
\begin{aligned}
& \int d \mu\left(p^{\prime}\right) d \mu\left(q^{\prime}\right) d \mu(p) d \mu(q) \delta^{d}\left(p+q-p^{\prime}-q^{\prime}\right) \\
& \varphi^{\prime}\left(p^{\prime}\right) \overline{\psi^{\prime}\left(q^{\prime}\right)}<p^{\prime}, q^{\prime}|T| p, q>\varphi(p) \psi(q)=0
\end{aligned}
$$

for each $\varphi, \psi, \psi^{\prime} \in \Phi^{(1)}$ with support in $R_{p}, R_{q}, R_{q^{\prime}}$ respectively. Suppose, by contradiction, that $p_{0}^{\prime}$ is in $\operatorname{supp}\left(\varphi^{\prime}\right)$. This implies that

$$
<p_{0}^{\prime}, p+q-p_{0}^{\prime}|T| p, q>=0
$$

whenever $p \in R_{p}, q \in R_{q}$ and $p+q-p_{0}^{\prime} \in R_{q^{\prime}}$. This region corresponds to an open set in the $(s, t)$ plane, so the analyticity of $\left\langle T^{(2,2)}\right\rangle$ (assumption 6 of the theorem) implies that it vanishes for any momenta in $\hat{\mathcal{T}}_{m}$. In particular, the elastic scattering amplitudes vanish for particles with mass $m$, in contradiction to assumption 7 of the theorem.

So $p^{\prime}$ is not in the support of $\varphi^{\prime}$. Since $p^{\prime}$ is arbitrary, this means that $\varphi^{\prime}=0$, i.e. $\chi$ is annihilated by $f \cdot A$ and since $\chi$ is arbitrary, this means that $f \cdot A=0$.

Finally, summarizing this part, we have:

\section{Proposition 1:}

The momentum support of $\langle A\rangle$ for any $A \in \mathcal{A}$ is restricted to the submanifold of $\hat{\mathcal{T}}_{F} \times \hat{\mathcal{T}}_{F}$ determined by the constraint $p^{\prime}=p$.

Proof:

Lemma 5 showed that for each $0 \neq \Delta p \in \hat{\mathcal{T}}$ there is a neighborhood $\Delta R$ of $\Delta p$ such that for each $f \in \mathcal{D}(\hat{\mathcal{T}})$ with support in $\Delta R, f \cdot A=0$. Since

$$
<p|f \cdot A| p^{\prime}>=f\left(p-p^{\prime}\right)<p|A| p^{\prime}>
$$

this means that $A$ vanishes (as a distribution) at the region

$$
\left\{\left(p, p^{\prime}\right) \in \hat{\mathcal{T}}_{F} \times \hat{\mathcal{T}}_{F} \mid p-p^{\prime} \in \Delta R\right\}
$$

In particular, if $p^{\prime}-p=\Delta p$ then $\left(p, p^{\prime}\right)$ is not in the support of $\langle A>$ and this is true for any $\Delta p \neq 0$. 


\section{Part 2}

This part analyzes the structure of the elements of

$$
\mathcal{B}:=\{B \in \mathcal{A} \mid B \text { is self adjoint and }[P, B]=0\}
$$

where $[P, B]:=P^{\prime} B-B P$ is an element of $L^{\times}\left(\Phi^{(1)} ; \Phi^{(1)^{\prime}}\right)$

( $P^{\prime}$ is the dual of $P$ and is an element of $L\left(\Phi^{\prime}\right)$ ).

$$
<p^{\prime}|[P, B]| p>=\left(p^{\prime}-p\right)<p^{\prime}|B| p>
$$

thus for each self adjoint element $B$ of $\mathcal{A}$ :

$$
B \in \mathcal{B} \Longleftrightarrow<p^{\prime}|B| p>=\delta\left(p^{\prime}-p\right) B(p)
$$

where $B(p)$ is a generalized function on the submanifold of $\Omega \times \Omega$, characterized by the constraint $p^{\prime}=p$ [16, vol. 1, p. 209], which is a matrix-valued generalized function on $\hat{\mathcal{T}}_{F}$. As an operator (from $\Phi^{(1)}$ to $\left.\Phi^{(1)^{\prime}}\right) B \in \mathcal{B}$ acts by multiplication:

$$
(B \varphi)(p)=B(p) \varphi(p) \forall \varphi \in \Phi^{(1)}
$$

(notice that this is a matrix multiplication, $B(p)$ being a Hermitian matrix).

Most of the analysis will be performed on elements of

$$
\mathcal{B}^{\infty}:=\left\{B \in \mathcal{B} \mid B \text { is a smooth function of } p \in \hat{\mathcal{T}}_{F}\right\}
$$

For each $p \in \hat{\mathcal{T}}_{F}$ and $B \in \mathcal{B}^{\infty}, B(p)$ is an operator in $\mathcal{H}(p)(N(p)$-dimensional matrix). According to eq. ([II.18), $B \in \mathcal{B}^{\infty}$ is a continuous operator in $\Phi^{(1)}$ and since it is self adjoint, it is extended continuously by its dual $B^{\prime}$ to $\Phi^{(1)^{\prime}}$. Its action on "plane waves" is:

$$
B^{\prime}|p>=| p>B(p)
$$

(recall from appendix A.6 that $\mid p>$ is a row vector)

\section{Lemma 1:}

$\mathcal{L}$ acts in $\mathcal{B}$ and $\mathcal{B}^{\infty}$ as a group of automorphisms.

For each $B \in \mathcal{B},(\Lambda, a) \in \mathcal{P}$,

$$
\left[U(\Lambda, a)^{\prime} B U(\Lambda, a)\right](p)=L^{(p)}\left(\Delta\left(\Lambda, p^{\prime}\right)\right)^{\dagger} B\left(p^{\prime}\right) L^{(p)}\left(\Delta\left(\Lambda, p^{\prime}\right)\right)
$$

where $p^{\prime}:=\Lambda p$.

(Throughout this Section, "†" denotes the Hermitian conjugation of (matrix) maps between the spaces $\mathcal{H}\left(p^{n}\right)$.) This follows directly from the expression for $\left\langle\psi\left|U(\Lambda, a)^{\prime} B U(\Lambda, a)\right| \varphi>\right.$ $\left(\varphi, \psi \in \Phi^{(1)}\right)$, using also the $\mathcal{L}$-invariance of $\mu$. 


\section{The Traceless Parts}

Let $B \in \mathcal{B}^{\infty}, p \in \hat{\mathcal{T}}_{F}$. The traceless part of $B(p)$ is

$$
B^{*}(p)=B(p)-\frac{1}{N(p)} 1(p) \operatorname{tr} B(p)
$$

where (see appendix A.4 for review of notation)

- $N(p)$ is the dimension of $\mathcal{H}(p)$;

- $1(p)$ is the identity operator in $\mathcal{H}(p)$;

- the trace $\operatorname{tr} B(p)$ of $B(p)$ is:

$$
\operatorname{tr} B(p):=\sum_{[\alpha, \lambda] \in \Omega(p)} B_{[\alpha, \lambda][\alpha, \lambda]}(p)
$$

(Notice that throughout this Section, “*” does not denote the Hilbert-space-adjoint). Let $\mathcal{B}^{*}$ denote the set of all traceless parts of elements of $\mathcal{B}^{\infty}$ :

$$
\mathcal{B}^{*}:=\left\{B^{*} \mid B \in \mathcal{B}^{\infty}\right\}
$$

\section{Lemma 2:}

$\mathcal{L}$ acts on $\mathcal{B}^{*}$ as a group of automorphisms: for each $\Lambda \in \mathcal{L}, B \in \mathcal{B}^{\infty}$

$$
U(\Lambda)^{\prime} B^{*} U(\Lambda)=\left[U(\Lambda)^{\prime} B U(\Lambda)\right]^{*}
$$

Proof:

Using eq. (III.21) we obtain:

$$
\begin{aligned}
& \operatorname{tr}\left[\left(U(\Lambda)^{\prime} B U(\Lambda)\right)(p)\right]= \\
& \quad=\operatorname{tr}\left[L^{(p)}(\Delta(\Lambda, \Lambda p))^{\dagger} B(\Lambda p) L^{(p)}(\Delta(\Lambda, \Lambda p))\right]=\operatorname{tr}[B(\Lambda p)]
\end{aligned}
$$

therefore, using eq. ([I.22), we have

$$
\begin{aligned}
& {\left[U(\Lambda)^{\prime} B^{*} U(\Lambda)\right](p)=} \\
& \quad=L^{(p)}(\Delta(\Lambda, \Lambda p)) B^{*}(\Lambda p) L^{(p)}(\Delta(\Lambda, \Lambda p))=\left[U(\Lambda)^{\prime} B U(\Lambda)\right]^{*}(p)
\end{aligned}
$$

In the next subsection it will be shown that this action is trivial. To do this we show now that $\mathcal{B}^{*}$ is a Lie algebra of matrices. We say that $p^{2} \in \hat{\mathcal{T}}_{F}^{2}$ is a null pair if there exists $0 \neq h \in \mathcal{H}\left(p^{2}\right)$, such that $<q^{2}|T| p^{2}>h$ vanishes as a function of $q^{2} \in \hat{\mathcal{T}}_{F}^{2}$ (in physicists' terminology this implies that "plane waves" with this combination of momenta, spins and particle types do not scatter elastically). We now show that $B^{*}$ is determined by its value 
for one non-null pair (and therefore $\mathcal{B}^{*}$ is, indeed, a Lie algebra of matrices). This will be shown by stages, in the next four lemmas.

As an element of $\mathcal{A}, B$ acts on two-particle states according to:

$$
B \varphi=(B \otimes I+I \otimes B) \varphi, \forall \varphi \in \Phi^{(1)} \times \Phi^{(1)}
$$

therefore

$$
(B \varphi)(p, q)=B(p, q) \varphi(p, q)
$$

where

$$
B(p, q):=B(p) \otimes 1(q)+1(p) \otimes B(q)
$$

The trace of $B(p, q)$ is

$$
\operatorname{tr} B(p, q)=N(q) \operatorname{tr} B(p)+N(p) \operatorname{tr} B(q)
$$

therefore the traceless part $B^{*}(p, q)$ of $B(p, q)$ is

$$
\begin{aligned}
B^{*}(p, q) & =B(p, q)-\frac{1}{N(p) N(q)} 1(p) \otimes 1(q) \operatorname{tr} B(p, q) \\
& =B^{*}(p) \otimes 1(q)+1(p) \otimes B^{*}(q) .
\end{aligned}
$$

which means that

$$
B^{*}(p, q)=0 \Longleftrightarrow B^{*}(p)=0 \text { and } B^{*}(q)=0
$$

\section{Lemma 3:}

If for $p, q \in \hat{\mathcal{T}}_{F},(p, q)$ is a non-null pair and $B^{*}(p, q)=0$ then for each $\Lambda \in \mathcal{L}(p+q), B^{*}(\Lambda p, \Lambda q)=0$

Proof:

Let $\Lambda \in \mathcal{L}(p+q)$ (a "rotation" in the "center of mass" of $(p, q))$. We denote $p^{\prime}:=\Lambda p, \quad q^{\prime}:=\Lambda q$. Since $B^{*}(p, q)=0, B(p, q)$ is a scalar matrix so it commutes with $L^{(p)}\left(\Delta\left(\Lambda, p^{\prime}\right)\right) \otimes L^{(q)}\left(\Delta\left(\Lambda, q^{\prime}\right)\right)$. Thus eq. (III.21) reduces in this case to

$$
B\left(p^{\prime}, q^{\prime}\right)=\left[U\left(\Lambda^{-1}\right) B U(\Lambda)\right](p, q)
$$

As an element of a Lie group, $\Lambda$ belongs to some one-parameter subgroup $\Lambda(\theta)$ of $\mathcal{L}(p+q)$ (analytic in $\theta$ ) which is generated in $\Phi^{(1)}$ by a continuous operator $J$ (see appendix A.8):

$$
U(\Lambda(\theta)) \varphi=e^{i \theta J} \varphi, \forall \varphi \in \Phi^{(1)} .
$$

A Taylor expansion in $\theta$ gives:

$$
U\left(\Lambda(\theta)^{-1}\right) B U(\Lambda(\theta))=e^{-i \theta J} B e^{i \theta J}=\sum_{n=0}^{\infty} \frac{\theta^{n}}{n !} \mathcal{F}_{J}^{n}(B)
$$


(the left hand side is a holomorphic (operator valued) function of $\theta$, thus the right hand side converges absolutely for all $\theta$ ) where $\mathcal{F}_{J}$ is an operator on $L\left(\Phi^{(1)}\right)$ defined by

$$
\mathcal{F}_{J}(C):=i[J, C], \forall C \in L\left(\Phi^{(1)}\right)
$$

In the following we prove that if $C \in \mathcal{B}^{\infty}$ satisfies $C^{*}(p, q)=0$ then so does $\mathcal{F}_{J}(C)$. This implies, by induction, that for each integer $n,\left[\mathcal{F}_{J}^{n}(B)\right](p, q)$ is scalar. Combining eqs. ([II.32) and ([III.34) we conclude that $B\left(p^{\prime}, q^{\prime}\right)$ is scalar, which means that $B^{*}\left(p^{\prime}, q^{\prime}\right)=0$, as claimed. So it is left to show that $[J, B]^{*}(p, q)=0$.

$B(\Lambda(\theta) p) \equiv\left(e^{-i \theta J} B e^{i \theta J}\right)(p)$ is a smooth function of $\theta$ and $p$ so

$$
i[J, B](p)=\left.\frac{d}{d \theta}\left(e^{-i \theta J} B e^{i \theta J}\right)\right|_{\theta=0}
$$

is also smooth in $p$. Since $B$ and $J$ are self adjoint, so is $i[J, B]$ and according to property 4 of $\mathcal{A}, i[J, B]$ is an element of $\mathcal{A}$. So we can conclude that $i[J, B] \in \mathcal{B}^{\infty}$.

Suppose, that $B^{*}\left(p^{\prime}, q^{\prime}\right) \neq 0 . B\left(p^{\prime}, q^{\prime}\right)$ is diagonalizable (since it is Hermitian) and since it is not scalar, it has at least two different eigenvalues, so at least one of them $b^{\prime}$ is different from the (unique) eigenvalue $b$ of $B(p, q)$. Let $h \in \mathcal{H}(p, q)$ be an eigenvector of $B\left(p^{\prime}, q^{\prime}\right)$ belonging to $b^{\prime} . B$ commutes with $S$ (as a self adjoint element of $\boldsymbol{\mathcal { A }}$ ) so we have (using eq. (III.20)

$$
\begin{aligned}
0 & =h^{\dagger}<p^{\prime}, q^{\prime}|[B, S]| p, q>h= \\
& =\left[B\left(p^{\prime}, q^{\prime}\right) h\right]^{\dagger}<p^{\prime}, q^{\prime}|S| p, q>h-h^{\dagger}<p^{\prime}, q^{\prime}|S| p, q>[B(p, q) h]= \\
& =\left(b^{\prime}-b\right) h^{\dagger}<p^{\prime}, q^{\prime}|S| p, q>h .
\end{aligned}
$$

For $\Lambda \neq 1$ this becomes

$$
h^{\dagger}<p^{\prime}, q^{\prime}|T| p, q>h=0
$$

(Here we use the fact that $\Lambda$ is in $\mathcal{L}(p+q)$ which means that $p^{\prime}+q^{\prime}=p+q$ ) so the assumption that $B^{*}\left(p^{\prime}, q^{\prime}\right) \neq 0$ leads to eq. (III.36). We are going to show that eq. ([11.36) cannot be satisfied for $\Lambda=1$. This implies, since the left-hand side of ([II.36) is continuous in $\theta$, that ([II.36) is not satisfied also in some neighborhood of the identity so in this neighborhood $B^{*}\left(p^{\prime}, q^{\prime}\right)=0$.

$$
\begin{aligned}
B\left(p^{\prime}, q^{\prime}\right) & =\left(e^{-i \theta J} B e^{i \theta J}\right)(p, q)= \\
& =B(p, q)+i \theta[J, B](p, q)+\mathcal{O}\left(\theta^{2}\right)
\end{aligned}
$$

Since $B^{*}(p, q)=0$ and for $\theta$ sufficiently small $B^{*}\left(p^{\prime}, q^{\prime}\right)=0$, we have $[J, B]^{*}(p, q)=0$.

Finally we must show that eq. ([II.36) cannot be satisfied for $\Lambda=1$, i.e.

$$
h^{\dagger}<p^{2}|T| p^{2}>h \neq 0
$$


for any $0 \neq h \in \mathcal{H}\left(p^{2}\right)$, where $p^{2}=(p, q)$. The expression

$$
h^{\dagger}<p^{2}|T| q^{m}>^{\dagger}<q^{m}|T| p^{2}>h
$$

is non negative (being the norm of $<q^{m}|T| p^{2}>h$ ). Since $p^{2}$ is non null, there exists $q_{0}^{2} \in \hat{\mathcal{T}}_{F}^{2}$ for which $<q_{0}^{2}|T| p^{2}>h \neq 0$, and since $<T^{(2,2)}>$ is analytic, the expression ([II.38) is positive in some neighborhood of $q_{0}^{2}$. Using the optical theorem (see appendix A.9) we, therefore, obtain

$$
\begin{aligned}
0 & <\int_{\hat{\mathcal{T}}_{F}^{m_{0}}} d \mu^{2}\left(q^{2}\right)(2 \pi)^{d} \delta^{d}\left(q_{1}+q_{2}-p_{1}-p_{2}\right) h^{\dagger}<p^{2}|T| q^{2}>^{\dagger}<q^{2}|T| p^{2}>h \\
& \leq \frac{1}{i} h^{\dagger}\left(<p^{2}|T| p^{2}>-<p^{2}|T| p^{2}>^{\dagger}\right) h \\
& =2 \operatorname{Im}\left(h^{\dagger}<p^{2}|T| p^{2}>h\right) .
\end{aligned}
$$

For $p_{1}, p_{2} \in \hat{\mathcal{T}}_{m}$, let $S\left(p_{1}, p_{2}\right)$ be the set of all the space-like parts of elements of $\hat{\mathcal{T}}_{m}$ that can be transformed to $p_{1}$ by an element of $\mathcal{L}\left(p_{1}+p_{2}\right)$. If $p_{1}+p_{2}=0$ then $\mathcal{L}\left(p_{1}+p_{2}\right)$ is the rotation group so $S\left(p_{1}, p_{2}\right)$ is a sphere around the origin containing $\vec{p}_{1}$ and $\vec{p}_{2}$. In general, $S\left(p_{1}, p_{2}\right)$ is the transformation of such a sphere by the $\mathcal{L}$-transformation $\Lambda_{p_{1}+p_{2}}$, and it can be shown that it is a $\left(r-1\right.$ dimensional) ellipsoid, symmetric around $\vec{p}_{1}+\vec{p}_{2}$ and longer in this direction then in the other ones.

\section{Lemma 4:}

If for $p_{1}, p_{2} \in \hat{\mathcal{T}}_{m},\left(p_{1}, p_{2}\right)$ is non-null and $B^{*}\left(p_{1}, p_{2}\right)=0$ then for each $q_{1} \in S\left(p_{1}, p_{2}\right), B^{*}\left(q_{1}\right)=0$.

Proof:

By definition, $q_{1} \in S\left(p_{1}, p_{2}\right)$ iff $q_{1} \in \hat{\mathcal{T}}_{m}$ and there exists $q_{2} \in \hat{\mathcal{T}}_{m}$ for which $q_{1}+q_{2}=p_{1}+p_{2}$ ( $q_{2}$ is the momentum transformed to $p_{2}$ by the element of $\mathcal{L}\left(p_{1}+p_{2}\right)$ which transforms $q_{1}$ to $\left.p_{1}\right)$. Since $\left(p_{1}, p_{2}\right)$ is non-null and $B^{*}\left(p_{1}, p_{2}\right)=0$, lemma 3 implies that $B^{*}\left(q_{1}, q_{2}\right)=0$ so according to relation (III.31), $B^{*}\left(q_{1}\right)=0$.

\section{Lemma 5:}

If, for $r>0$, each $p \in \hat{\mathcal{T}}_{m}$ with $|\vec{p}|=r$ satisfies $B^{*}(p)=0$ then there exists $r^{\prime}>r$ such that for each $p^{\prime} \in \hat{\mathcal{T}}_{m}$ which satisfies $\left|\vec{p}^{\prime}\right| \leq r^{\prime}, B^{*}\left(p^{\prime}\right)=0$.

Proof:

We construct $p_{1}, p_{2} \in \hat{\mathcal{T}}_{m}$ with $\left|\vec{p}_{1}\right|=\left|\vec{p}_{2}\right|=r$ for which $p_{m}=(m, 0, \ldots, 0)$ is in $S\left(p_{1}, p_{2}\right)$. This requirement is equivalent to

$$
p_{1}+p_{2}-p_{m} \in \hat{\mathcal{T}}_{m}
$$


which gives a condition on the angle $\theta$ between $\vec{p}_{1}$ and $\vec{p}_{2}$ :

$$
2 \sqrt{m^{2}+r^{2}}=m+\sqrt{m^{2}+4 r^{2} \cos ^{2} \frac{\theta}{2}} .
$$

This equation has a (unique positive) solution for $\theta$ which is an increasing function of $\frac{r}{m}$. Thus the required momenta exist and they satisfy $\vec{p}_{1} \neq \vec{p}_{2} \neq-\vec{p}_{1}(0 \neq \theta \neq \pi)$.

Now define

$$
r^{\prime}:=\max \left\{\left|\vec{p}^{\prime}\right| \mid \vec{p}^{\prime} \in S\left(p_{1}, p_{2}\right)\right\}
$$

(the maximum exists since $\mathcal{L}\left(p_{1}+p_{2}\right)$ is compact and acts continuously in $\left.\hat{\mathcal{T}}_{m}\right)$. Considering the shape of $S\left(p_{1}, p_{2}\right)$, the fact that $\vec{p}_{1} \neq \vec{p}_{2}$ implies that $S\left(p_{1}, p_{2}\right)$ is not contained entirely inside this sphere which means that $r^{\prime}>r . S\left(p_{1}, p_{2}\right)$ is connected (this comes from the connectedness of $\mathcal{L}\left(p_{1}+p_{2}\right)$ ) thus it contains momenta with any magnitude in the range $\left[0, r^{\prime}\right]$.

Let $p^{\prime} \in \hat{\mathcal{T}}_{m}$ for which $\left|\vec{p}^{\prime}\right| \leq r^{\prime}$. One can always perform a rotation around the origin, transforming $p_{1}$ and $p_{2}$ to put $\vec{p}^{\prime}$ in $S\left(p_{1}, p_{2}\right)$. This rotation does not change the magnitude of $\vec{p}_{1}$ and $\vec{p}_{2}$ so we still have $B^{*}\left(p_{1}\right)=B^{*}\left(p_{2}\right)=0$ which means (by eq. ([II.31)) that $B^{*}\left(p_{1}, p_{2}\right)=0$. If $\left(p_{1}, p_{2}\right)$ is non-null then lemma 4 implies that $B^{*}\left(p^{\prime}\right)=0$. If $\left(p_{1}, p_{2}\right)$ is null, a slight deformation of $p_{2}$ preserving $\left|\vec{p}_{2}\right|=r$ can be made to change $s=\left(p_{1}+p_{2}\right)^{2}$ and since null pairs exist only for isolated values of $(s, t)$ (according to assumptions 6 and 7 ), we will get a non-null pair. This implies that $p^{\prime}$ is on a boundary of a region in which $B^{*}$ vanishes so, by the continuity of $B^{*}$, we obtain $B^{*}\left(p^{\prime}\right)=0$.

\section{Lemma 6:}

If $p, q \in \hat{\mathcal{T}}_{m}$ are different and such that $(p, q)$ is non-null and $B^{*}(p, q)=0$ then $B^{*}$ vanishes on $\hat{\mathcal{T}}_{m}$.

Proof:

First assume $\vec{p}+\vec{q}=0$ and define

$$
r:=\sup \left\{r^{\prime} \mid B^{*}\left(p^{\prime}\right)=0, \text { for all } p^{\prime} \in \hat{\mathcal{T}}_{m} \text { with }\left|\vec{p}^{\prime}\right| \leq r^{\prime}\right\} \text {. }
$$

Lemma 4 implies that for each $p^{\prime} \in \hat{\mathcal{T}}_{m}$ with $\left|\vec{p}^{\prime}\right|=|\vec{p}|, B^{*}\left(p^{\prime}\right)=0$, so, by lemma $5, r \geq|\vec{p}|>0$.

Assume, by contradiction, that $r$ is finite. By definition, for each $p^{\prime} \in \hat{\mathcal{T}}_{m}$ with $\left|\vec{p}^{\prime}\right|<r, B^{*}\left(p^{\prime}\right)=0$ so if $p^{\prime} \in \hat{\mathcal{T}}_{m}$ satisfies $\left|\vec{p}^{\prime}\right|=r$, it is on the boundary of a region in which $B^{*}$ vanishes, thus, by continuity, $B^{*}\left(p^{\prime}\right)=0$. Looking back at lemma 5 one observes that it contradicts the maximality of $r$. Therefore $r=\infty$ which means that $B^{*}$ vanishes on $\hat{\mathcal{T}}_{m}$. 
Returning to the general case (where $\vec{p}+\vec{q} \neq 0$ ), define:

$$
p^{\prime}:=\Lambda_{p+q}^{-1} p, q^{\prime}:=\Lambda_{p+q}^{-1} q, B^{\prime}:=U\left(\Lambda_{p+q}^{-1}\right) B U\left(\Lambda_{p+q}\right)
$$

(this is a transformation to the "rest frame" of $p+q$ ). Eq. (III.31) implies that $B(p)$ and $B(q)$ are scalars thus commute with $L^{(p)}$. Therefore, eq. (III.21) gives

$$
B^{\prime}\left(p^{\prime}\right)=L^{(p)}\left(\Delta\left(\Lambda_{p+q}, p\right)\right)^{\dagger} B(p) L^{(p)}\left(\Delta\left(\Lambda_{p+q}, p\right)\right)=B(p)
$$

and in the same way $B^{\prime}\left(q^{\prime}\right)=B(q)$, thus

$$
B^{\prime *}\left(p^{\prime}, q^{\prime}\right)=B^{*}(p, q)=0
$$

The pair $\left(p^{\prime}, q^{\prime}\right)$ is non-null since it was obtained from a non-null pair by an $\mathcal{L}$-transformation which doesn't change the S-matrix; therefore $B^{\prime}$ satisfies the assumptions of the lemma and $\vec{p}^{\prime}+\vec{q}^{\prime}=0$, so the first part implies that $B^{\prime *}=0$. Since $B^{*}=U\left(\Lambda_{p+q}\right) B^{\prime *} U\left(\Lambda_{p+q}^{-1}\right)$ (according to lemma 2) this means that also $B^{*}=0$.

¿From the property 4 of $\mathcal{A}$ and the definitions of $\mathcal{B}, \mathcal{B}^{\infty}$, and $\mathcal{B}^{*}$, it follows that $\mathcal{B}^{*}$ is a real Lie algebra. If $B_{1}$ and $B_{2}$ are in $\mathcal{B}^{\infty}$ then so is $i\left[B_{1}, B_{2}\right]$ and

$$
i\left[B_{1}^{*}, B_{2}^{*}\right]=i\left[B_{1}, B_{2}\right]^{*} \in \mathcal{B}^{*} .
$$

Denote by $\mathcal{B}_{m}^{*}$ the set of restrictions of elements of $\mathcal{B}^{*}$ to one orbit $\hat{\mathcal{T}}_{m}(m \in \mathcal{M})$. This is a real Lie algebra of smooth functions from $\hat{\mathcal{T}}_{m}$ to $\mathcal{H}(m)$. For $B^{*} \in \mathcal{B}_{m}^{*}, p \in \hat{\mathcal{T}}_{m}, B^{*}(p)$ is a traceless Hermitian $N(m) \times N(m)$ dimensional matrix which is an element of the Lie algebra $s u(N(m))$ of the group $S U\left(N(m)\right.$. Therefore, for $p, q \in \hat{\mathcal{T}}_{m}, B^{*}(p, q)$ can be identified as an element of $s u(N(m)) \oplus s u(N(m))$ which is a finite dimensional compact Lie algebra. The mapping

$$
B^{*} \mapsto B^{*}(p, q)
$$

is a homomorphism for the Lie algebra structure and lemma 6 implies that if $(p, q)$ is nonnull, this homomorphism is an isomorphism (injective) which implies (since non-null pairs exist) that $\mathcal{B}_{m}^{*}$ can be identified as a subalgebra of a compact Lie algebra (of matrices). As such, its structure is

$$
\mathcal{B}_{m}^{*}=\mathcal{B}_{s} \oplus \mathcal{B}_{c} \text { (a direct sum of ideals) }
$$

where

$\mathcal{B}_{s}$ is a semisimple compact Lie algebra;

$\mathcal{B}_{c}$ is the center of $\boldsymbol{\mathcal { B }}_{m}^{*}$, an abelian Lie algebra. 


\section{The Action of $\mathcal{L}$ on the Traceless Parts}

According to lemmas 1 and $2, \mathcal{L}$ acts on $\mathcal{B}_{m}^{*}$ as a group of automorphisms and this action preserves the commutator:

$$
\left[U^{-1} B_{1} U, U^{-1} B_{2} U\right]=U^{-1}\left[B_{1}, B_{2}\right] U, \forall B_{1}, B_{2} \in \mathcal{B}_{m}^{*} .
$$

We use this to prove:

\section{Lemma 7:}

$\mathcal{B}_{s}$ and $\mathcal{B}_{c}$ are $\mathcal{L}$-invariant

Proof:

For sets $S_{1}, S_{2} \subset \mathcal{B}_{m}^{*}$, denote $\left[S_{1}, S_{2}\right]$ the real vector space spanned by

$$
\left\{\left[B_{1}, B_{2}\right] \mid B_{1} \in S_{1}, B_{2} \in S_{2}\right\} .
$$

$U \mathcal{B}_{m}^{*} U^{-1}=\mathcal{B}_{m}^{*}$ and $\left[\mathcal{B}_{c}, \mathcal{B}_{m}^{*}\right]=0$ so

$$
\left[U^{-1} \mathcal{B}_{c} U, \mathcal{B}_{m}^{*}\right]=U^{-1}\left[\mathcal{B}_{c}, U \mathcal{B}_{m}^{*} U^{-1}\right] U=0
$$

thus $U^{-1} \mathcal{B}_{c} U \subset \mathcal{B}_{c}$ i.e. $\mathcal{B}_{c}$ is $\mathcal{L}$-invariant.

$\left[\mathcal{B}_{c}, \mathcal{B}_{m}^{*}\right]=0$ implies that $\left[\mathcal{B}_{m}^{*}, \mathcal{B}_{m}^{*}\right]=\left[\mathcal{B}_{s}, \mathcal{B}_{s}\right]$ and the semisimplicity of $\boldsymbol{B}_{s}$ implies that $\left[\mathcal{B}_{s}, \mathcal{B}_{s}\right]=\mathcal{B}_{s}$; therefore

$$
\begin{aligned}
U^{-1} \mathcal{B}_{s} U & =U^{-1}\left[\mathcal{B}_{m}^{*}, \mathcal{B}_{m}^{*}\right] U=\left[U^{-1} \mathcal{B}_{m}^{*} U, U^{-1} \mathcal{B}_{m}^{*} U\right]= \\
& =\left[\mathcal{B}_{m}^{*}, \mathcal{B}_{m}^{*}\right]=\mathcal{B}_{s},
\end{aligned}
$$

which means that $\mathcal{B}_{s}$ is $\mathcal{L}$-invariant.

Lemma 7 means that $\mathcal{L}$ acts as a group of automorphisms in each of the ideals $\boldsymbol{B}_{s}$ and $\mathcal{B}_{c}$. The representation of $\mathcal{L}$ (as a group of automorphisms) is a homomorphism so its kernel is a normal subgroup of $\mathcal{L}$. $\mathcal{L}_{0}$ is a simple group which means that it doesn't have non-trivial normal subgroups (that is other than $\mathcal{L}_{0}$ itself and $\{e\}$, where $e$ is the identity in $\left.\mathcal{L}_{0}\right) . \mathcal{L}_{0}=\mathcal{L} / \mathbf{Z}_{2}$ (since $r \geq 3$; see appendix A.1), Therefore $\mathbf{Z}_{2}$ is the only non trivial normal subgroup of $\mathcal{L}$. To show that $\mathcal{L}$ is represented trivially (the kernel is $\mathcal{L}$ ) it is enough to show that the kernel is not contained in $\mathbf{Z}_{2}$. This will be done in the next two lemmas.

\section{Lemma 8:}

$\mathcal{L}$ acts trivially in $\boldsymbol{B}_{s}$.

Proof:

The connected part of the group of automorphisms of a compact semisimple Lie algebra is known to be the corresponding compact semisimple group, so the representation of $\mathcal{L}$ in $\mathcal{B}_{s}$ is a homomorphism from $\mathcal{L}$ to a compact group. Since $\mathcal{L}$ and $\mathcal{L}_{0}=\mathcal{L} / \mathbf{Z}_{2}$ are not compact and therefore cannot be contained in a compact group, the kernel must be all of $\mathcal{L}$. 


\section{Lemma 9:}

$\mathcal{L}$ acts trivially in $\boldsymbol{B}_{c}$.

Proof:

We choose $p, q \in \hat{\mathcal{T}}_{m}$ such that $(p, q)$ is non-null and $\vec{p}+\vec{q}=0$ (so that $\mathcal{L}(p+q)=\mathcal{O}_{0}(r)$, the connected part of $\left.\mathcal{O}(r)\right)$. Since $r \geq 3, \mathcal{L}(p+q)$ contains a one-parameter subgroup of rotations $R(\theta)$ around $\vec{p}$. $R(\theta)$ doesn't change $p$ and $q$ therefore it is represented in $\mathcal{B}_{m}^{*}$ by $L^{(p)} \otimes L^{(q)}$ acting on the matrices $B^{*}(p, q) . R(\theta)$ is abelian thus its (finite dimensional) irreducible representations in a complex Hilbert space are one-dimensional. Let $B$ be an element of the complex extension of $\boldsymbol{B}_{c}$ which transforms irreducibly under $R(\theta)$. In the following we will show that if $B$ doesn't transform trivially then $B$ cannot commute with its adjoint, in contradiction to the commutativity of $\boldsymbol{B}_{c}$. To show this, we need an explicit representation for the matrix $B(p, q)$. Let $J$ be the generator of $\left(L^{(p)} \otimes L^{(q)}\right)(R(\theta))$. This is a Hermitian matrix (since $L^{(p)}$ is unitary) thus diagonalizable, so we assume that the diagonalization has been performed. In this basis, $B(p, q)$, abbreviated in the following by $B$, can be seen as a block matrix $B=\left\{B_{i j}\right\}$, where each block $B_{i j}$ connects the eigenspaces of the eigenvalues $i$ and $j$ of $J$. The action of $R(\theta)$ on $B$ is

$$
B \stackrel{R(\theta)}{\longmapsto} e^{-i \theta J} B e^{i \theta J}=e^{-i \theta k} B
$$

where $\mathrm{k}$ is some real number. By optionally switching $B$ with $B^{\dagger}$ one can always have $k \geq 0$ so we assume that it is so. Differentiating eq. (III.50), one obtains:

$$
[J, B]=k B .
$$

This means that B is a "ladder operator" for $J$ : if $h \in \mathcal{H}(p, q)$ satisfies $J h=j h$ then $J(B h)=(j+k)(B h)$, and this implies that $B_{i j}$ can be non-zero only if $i-j=k .\left(B^{\dagger}\right)_{i j}=\left(B_{j i}\right)^{\dagger}$ so we have:

$$
\begin{aligned}
\left(B B^{\dagger}\right)_{i j} & =\sum_{l} B_{i l}\left(B_{j l}\right)^{\dagger}=B_{i, i-k}\left(B_{j, j-k}\right)^{\dagger} \\
& =\delta_{i j} B_{i, i-k}\left(B_{j, j-k}\right)^{\dagger}
\end{aligned}
$$

and in the same way

$$
\left(B^{\dagger} B\right)_{i j}=\delta_{i j}\left(B_{i+k, i}\right)^{\dagger} B_{i+k, i} .
$$

Let $l$ be the maximal eigenvalue of $J$ for which $B_{l, l-k} \neq 0(\mathcal{H}(p, q)$ is finitedimensional and $B \neq 0$ so the maximum exists). This implies that $B_{l, l-k}\left(B_{l, l-k}\right)^{\dagger} \neq 0$ (since each element is a sum of squares). Now the commutativity of $\mathcal{B}_{c}$ implies that $B B^{\dagger}=B^{\dagger} B$ therefore eqs. (III.51) and ([II.52) give

$$
\left(B_{l+k, l}\right)^{\dagger} B_{l+k, l}=B_{l, l-k}\left(B_{l, l-k}\right)^{\dagger} \neq 0
$$


This means that $B_{l+k, l} \neq 0$ and the maximality of $l$ implies that $k=0$ which means that $B$ transforms trivially. Since $B$ is arbitrary, this implies that $J$ acts trivially which means that the kernel of the representation contains a one parameter group, which cannot be contained in $\mathbf{Z}_{2}$. Therefore the kernel is all of $\mathcal{L}$.

\section{The General Form of the Elements of $\mathcal{B}$}

The triviality of the action of $\mathcal{L}$ in $B^{*}$ (lemmas 7,8 and 9) implies that:

Lemma 10:

For each $B \in \mathcal{B}^{\infty}, p \in \hat{\mathcal{T}}_{m}$, the traceless part has the form

$$
B_{[\alpha \lambda]\left[\alpha^{\prime} \lambda^{\prime}\right]}^{*}=B_{\alpha \alpha^{\prime}}^{*} \delta_{\lambda \lambda^{\prime}}
$$

i.e. $B^{*}$ is independent of $p$ and it commutes with $L^{(p)}$.

Proof:

Lemmas 7,8 and 9 imply that for each $\Lambda \in \mathcal{L}$

$$
U\left(\Lambda^{-1}\right) B^{*} U(\Lambda)=B^{*}
$$

which means (by eq. (III.21)) that for each $p \in \hat{\mathcal{T}}_{m}$

$$
B^{*}(p)=L^{(p)}\left(\Delta\left(\Lambda, p^{\prime}\right)\right)^{\dagger} B^{*}\left(p^{\prime}\right) L^{(p)}\left(\Delta\left(\Lambda, p^{\prime}\right)\right) \quad, \quad\left(p^{\prime}=\Lambda p\right) .
$$

Recall (from appendix A.7.1) that

$$
\Delta(\Lambda, p)=\Lambda_{p}^{-1} \Lambda \Lambda_{\Lambda^{-1} p}
$$

where

$$
\Lambda_{p} p_{m}=p \quad, \quad \Lambda_{p_{m}}=1
$$

From this it follows that $\Delta\left(\Lambda_{p}, p\right)=1$. Using this in eq. (11.56) gives

$$
B^{*}\left(p_{m}\right)=L^{(p)}\left(\Delta\left(\Lambda_{p}, p\right)\right)^{\dagger} B^{*}(p) L^{(p)}\left(\Delta\left(\Lambda_{p}, p\right)\right)=B^{*}(p)
$$

therefore $B^{*}$ is independent of $p$. Eq. (III.57) also implies that

$$
\Delta\left(\Lambda, p_{m}\right)=\Lambda, \forall \Lambda \in \mathcal{L}\left(p_{m}\right)
$$

Using this in eq. (III.56) gives (since $B^{*}\left(p^{\prime}\right)=B^{*}(p)$ )

$$
\left[B^{*}, L^{(p)}(\Delta)\right]=0, \forall \Delta \in \mathcal{L}\left(p_{m}\right)
$$

and eq. ([II.54) follows from this by Schur's lemma.

\section{Lemma 11:}


For each $B \in \mathcal{B}^{\infty}$, if $p, q, p^{\prime}, q^{\prime} \in \hat{\mathcal{T}}_{m}$ satisfy $p+q=p^{\prime}+q^{\prime}$ then

$$
\operatorname{tr} B(p)+\operatorname{tr} B(q)=\operatorname{tr} B\left(p^{\prime}\right)+\operatorname{tr} B\left(q^{\prime}\right)
$$

which means that $\operatorname{tr} B(p)$ is a linear (real) function of $p$ :

$$
\operatorname{tr} B(p)=a_{\mu} p^{\mu}+b
$$

Proof:

Lemma 10 implies that

$$
B(p, q)=B^{*}(p, q)+\frac{1}{N(m)^{2}} 1(p, q) \operatorname{tr} B(p, q)
$$

where $B^{*}$ is some constant Hermitian matrix. Following a similar procedure as in the proof of lemma 3, we take some eigenvector $h \in \mathcal{H}(p, q)$ of $B^{*}$ belonging to some eigenvalue $b$. Eq. (III.63) implies that for each $p, q \in \hat{\mathcal{T}}_{m}, h$ is an eigenvector of $B$, with the eigenvalue

$$
b+\frac{1}{N(m)^{2}} \operatorname{tr} B(p, q)=b+\frac{1}{N(m)}(\operatorname{tr} B(p)+\operatorname{tr} B(q)) .
$$

Therefore we obtain

$$
\begin{aligned}
0 & =h^{\dagger}<p^{\prime}, q^{\prime}|[B, S]| p, q>h= \\
& =\frac{1}{N(m)}\left[\operatorname{tr} B\left(p^{\prime}\right)+\operatorname{tr} B\left(q^{\prime}\right)-\operatorname{tr} B(p)-\operatorname{tr} B(q)\right] h^{\dagger}<p^{\prime}, q^{\prime}|S| p, q>h
\end{aligned}
$$

and as in lemma 3, this implies that for a non-null pair, $\operatorname{tr} B(p)+\operatorname{tr} B(q)$ is locally $\mathcal{L}(p+q)$-invariant. Since $\mathcal{L}(p+q)$ is connected, this means global invariance and since each null pair is a limit of non-null pairs and $B(p)$ is continuous in $p$, this holds also for null pairs.

To extend these results from $\mathcal{B}^{\infty}$ to $\mathcal{B}$, we define, for each $B \in \mathcal{B}, f \in C_{c}^{\infty}(\mathcal{L})$

$$
B^{f}:=\int_{\mathcal{L}} d \Lambda f(\Lambda) U(\Lambda)^{\prime} B U(\Lambda)
$$

where $d \Lambda$ is the Haar measure of $\mathcal{L}$.

\section{Lemma 12:}

For each $B \in \mathcal{B}, f \in C_{c}^{\infty}(\mathcal{L}), B^{f}$ is an element of $\boldsymbol{B}^{\infty}$.

Proof: 
Let $\varphi, \psi \in \Phi^{(1)}$.

$$
\begin{aligned}
& \left(\psi \mid B^{f} \varphi>=\right. \\
& =\int_{\mathcal{L}} d \Lambda f(\Lambda) \int_{\hat{\mathcal{T}}_{F}} d \mu(p) \psi\left(\Lambda^{-1} p\right)^{\dagger} L^{(p)}(\Delta(\Lambda, p))^{\dagger} B(p) L^{(p)}(\Delta(\Lambda, p)) \varphi\left(\Lambda^{-1} p\right)= \\
& =\sum_{m \in \mathcal{M}} \int_{\hat{\mathcal{T}}_{m}} d \mu(p) \int_{\mathcal{L}} d\left(\Lambda_{p} \Lambda\right) f\left(\Lambda_{p} \Lambda\right) \\
& \quad \psi\left(\Lambda^{-1} p_{m}\right)^{\dagger} L^{(p)}\left(\Delta\left(\Lambda_{p} \Lambda, p\right)\right)^{\dagger} B(p) L^{(p)}\left(\Delta\left(\Lambda_{p} \Lambda, p\right)\right) \varphi\left(\Lambda^{-1} p_{m}\right)
\end{aligned}
$$

where $p_{m}=\Lambda_{p}^{-1} p$ (the last equality is due to Fubini's theorem for distributions and the fact that multiplication by $\Lambda_{p}$ is a bijection in $\mathcal{L}$ ). From eq. (A.23) we obtain

$$
\Delta\left(\Lambda_{p} \Lambda, p\right)=\Lambda \Lambda_{\Lambda^{-1} p_{m}} \text { (independent of } p \text { ) }
$$

therefore (using the $\mathcal{L}$-invariance of $d \Lambda$ and recalling that $L^{(p)}$ is determined by $m$ )

$$
\begin{aligned}
& \left(\psi \mid B^{f} \varphi>=\right. \\
& =\sum_{m \in \mathcal{M}} \int_{\mathcal{L}} d \Lambda \psi\left(\Lambda^{-1} p_{m}\right)^{\dagger} L^{(p)}\left(\Lambda \Lambda_{\Lambda^{-1} p_{m}}^{\dagger}\right) \\
& \quad\left[\int_{\hat{\mathcal{I}}_{m}} d \mu(p) B(p) f\left(\Lambda_{p} \Lambda\right)\right] L^{(p)}\left(\Lambda \Lambda_{\Lambda^{-1} p_{m}}\right) \varphi\left(\Lambda^{-1} p_{m}\right) \\
& =\sum_{m \in \mathcal{M}} \int_{\mathcal{L}} d \Lambda \psi\left(\Lambda p_{m}\right)^{\dagger} L^{(p)}\left(\Lambda^{-1} \Lambda_{\Lambda p_{m}}^{\dagger}\right) \\
& \quad\left[\int_{\hat{\mathcal{I}}_{m}} d \mu(p) B(p) f\left(\Lambda_{p} \Lambda^{-1}\right)\right] L^{(p)}\left(\Lambda^{-1} \Lambda_{\Lambda p_{m}}\right) \varphi\left(\Lambda p_{m}\right)
\end{aligned}
$$

Each $\Lambda \in \mathcal{L}$ can be decomposed uniquely to $\Lambda=\Lambda_{p} \Delta$ where $p=\Lambda p_{m}$ and $\Delta=\Lambda_{p}^{-1} \Lambda \in \mathcal{L}\left(p_{m}\right)$ therefore

$$
\int_{\mathcal{L}} d \Lambda \ldots=\int_{\hat{\mathcal{I}}_{m}} d \mu(p) \int_{\Lambda_{p} \mathcal{L}\left(p_{m}\right)} d \Lambda \ldots
$$

and this gives:

$$
\left(\psi \mid B^{f} \varphi>=\int_{\hat{\mathcal{T}}_{F}} d \mu(p) \psi(p)^{\dagger} B^{f}(p) \varphi(p)\right.
$$

where

$$
\begin{aligned}
& B^{f}(p)= \\
& \quad \int_{\Lambda_{p} \mathcal{L}\left(p_{m}\right)} d \Lambda L^{(p)}\left(\Lambda^{-1} \Lambda_{p}\right)^{\dagger}\left[\int_{\hat{\mathcal{T}}_{m}} d \mu\left(p^{\prime}\right) B\left(p^{\prime}\right) f\left(\Lambda_{p^{\prime}} \Lambda^{-1}\right)\right] L^{(p)}\left(\Lambda^{-1} \Lambda_{p}\right)
\end{aligned}
$$

Now the smoothness of $B^{f}(p)$ follows from the analyticity of $\mathcal{L}$ and the smoothness of $f, \Lambda_{p}$ and $L^{(p)}$.

Now it is possible to conclude with

\section{Proposition 2:}


On each orbit $\hat{\mathcal{T}}_{m}, B \in \mathcal{B}$ has the form

$$
B(p)=B+I a_{\mu} p^{\mu}
$$

where $B$ is a Hermitian matrix of the form

$$
B_{[\alpha \lambda]\left[\alpha^{\prime} \lambda^{\prime}\right]}=B_{\alpha \alpha^{\prime}} \delta_{\lambda \lambda^{\prime}}
$$

and $\left\{a_{\mu}\right\}$ is a real vector.

Proof:

For $B \in \mathcal{B}^{\infty}$ it is a direct result of lemmas 10 and 11 . Lemma 12 implies that

for each $f \in C_{c}^{\infty}(\mathcal{L}), B^{f}$ is in $\mathcal{B}^{\infty}$ so it has the form (III.68), therefore so does $B$.

\section{Part 3}

In this part, the results of the preceding parts (propositions 1 and 2) are combined to prove the statement of the theorem.

Let $A \in \mathcal{A}$. Proposition 1 states that the support of $\langle A\rangle$ is restricted to the submanifold of $\hat{\mathcal{T}}_{F} \times \hat{\mathcal{T}}_{F}$ defined by the constraint $p^{\prime}-p=0$. This implies [16, vol. 1] that $\langle A\rangle$ is a polynomial in the derivatives of $\delta\left(p^{\prime}-p\right)$. In other words, $\langle A\rangle$ is a differential operator (of finite order $N$ ):

$$
<p^{\prime}|A| p>=\delta\left(p^{\prime}-p\right) \sum_{n=0}^{N} A_{\vec{\mu}}^{(n)}(p) \frac{1}{i^{n}} \frac{\partial}{\partial p^{\mu_{1}}} \cdots \frac{\partial}{\partial p^{\mu_{n}}}
$$

where the coefficients $A_{\vec{\mu}}^{(n)}(p)$ are generalized (matrix valued) functions on $\hat{\mathcal{T}}_{F}$ and implicit summation over $\vec{\mu}=\left(\mu_{1}, \ldots, \mu_{n}\right)$ is assumed. Moreover, $\hat{\mathcal{T}}_{F}$ consists of isolated orbits (since the one-particle mass spectrum is finite), therefore the derivatives in $A$ are tangent to the orbits (which means that on each orbit $\hat{\mathcal{T}}_{m}, A$ is a polynomial in $\nabla_{\mu}=\frac{\partial}{\partial p^{\mu}}-\frac{p^{\mu} p^{\nu}}{m^{2}} \frac{\partial}{\partial p^{\nu}}$ ) and this implies that

$$
\left[A, P_{\mu} P^{\mu}\right]=0 .
$$

Combining eqs. ([11.69) and ([11.70) with proposition 2, we obtain:

\section{Lemma 1:}

For each self adjoint element $A$ of $\mathcal{A}, A_{\vec{\mu}}^{(N)}$ belongs to $\mathcal{B}$. As such, it has the form

$$
A_{\vec{\mu}}^{(N)}(p)=I a_{\nu \vec{\mu}} p^{\nu}+B_{\vec{\mu}}, B_{\vec{\mu}[\alpha \lambda]\left[\alpha^{\prime} \lambda^{\prime}\right]}=B_{\vec{\mu} \alpha \alpha^{\prime}} \delta_{\lambda \lambda^{\prime}}
$$

and if $N \geq 1$ then

$$
B_{\vec{\mu}}=0, a_{\nu \mu_{1} \mu_{2} \cdots \mu_{N}}=-a_{\mu_{1} \nu \mu_{2} \cdots \mu_{N}} .
$$

Proof: 
From $\left[A_{\vec{\mu}}^{(n)}, P_{\nu}\right]=0$ and $\left[\frac{\partial}{\partial p_{\mu}}, P_{\nu}\right]=\delta_{\nu}^{\mu}$ it follows that

$$
i\left[i\left[\ldots i\left[A, P_{\mu_{N}}\right] \ldots, P_{\mu_{2}}\right], P_{\mu_{1}}\right]=A_{\vec{\mu}}^{(N)} .
$$

Property 4 of $\mathcal{A}$ implies that $A_{\vec{\mu}}^{(n)}$ is in $\mathcal{A}$. $A$ and $P_{\mu}$ are Hermitian thus so is $A_{\vec{\mu}}^{(N)}$. Finally $\left[A_{\vec{\mu}}^{(N)}, P\right]=0$ therefore $A_{\vec{\mu}}^{(N)}$ is in $\mathcal{B}$ and proposition 2 gives its general form ([II.71).

For $N \geq 0$, property 4 of $\mathcal{A}$ implies that

$$
\left[\left[\ldots\left[A, P_{\mu_{N}}\right] \ldots, P_{\mu_{3}}\right], P_{\mu_{2}}\right] \in \mathcal{A}
$$

therefore, from eq. ([11.70) it follows that

$$
\begin{aligned}
0 & =i\left[i\left[\ldots i\left[A, P_{\mu_{N}}\right] \ldots, P_{\mu_{2}}\right], P_{\mu_{1}} P^{\mu_{1}}\right](p) \\
& =A_{\vec{\mu}}^{(N)}(p) p^{\mu_{1}}=I a_{\nu \vec{\mu}} p^{\nu} p^{\mu_{1}}+B_{\vec{\mu}} p^{\mu_{1}} .
\end{aligned}
$$

It can be shown that $\left\{p^{\nu} p^{\mu}\right\}$ and $\left\{p^{\mu}\right\}$ are all linearly independent functions on $\hat{\mathcal{T}}_{m}$ which means that eq. (III.72) follows from eq. (III.73).

Before stating the final result, one has to determine the form of the generators involved. The generators of $\mathcal{P}$ can be obtained by differentiating the explicit expression ( $\sqrt{\mathrm{A} .22}$ ) for the representation with respect to a parameter of a one-parameter subgroup of $\mathcal{P}$. The generators of an "internal" symmetry transformations are recognized by their commutativity with all elements of $\mathcal{P}$ (and their general form is determined using the methods described in part 2). This gives

\section{Lemma 2:}

- A generator of $\mathcal{L}$ is of the form

$$
A(p)=B(p)+I a_{\mu \nu} p^{\mu} \frac{1}{i} \frac{\partial}{\partial p_{\nu}}, B_{[\alpha \lambda]\left[\alpha^{\prime} \lambda^{\prime}\right]}(p)=\delta_{\alpha \alpha^{\prime}} B_{\lambda \lambda^{\prime}}^{\alpha}(p)
$$

where

$B(p)$ is a Hermitian matrix;

$\left\{a_{\mu \nu}\right\}$ is a real antisymmetric matrix $\left(a_{\mu \nu}=-a_{\nu \mu}\right)$.

- A generator of $\mathcal{T}$ is of the form

$$
A(p)=I a_{\mu} p^{\mu}
$$

where $\left\{a_{\mu}\right\}$ is a real vector.

- A generator of an internal symmetry is of the form

$$
A(p)=B, B_{[\alpha \lambda]\left[\alpha^{\prime} \lambda^{\prime}\right]}=B_{\alpha \alpha^{\prime}} \delta_{\lambda \lambda^{\prime}}
$$


Now it is possible to state and prove the final result:

\section{Proposition 3:}

A self adjoint element $A$ of $\mathcal{A}$ is a linear combination of generators of $\mathcal{P}$ and generators of internal symmetries.

Proof:

$N=0$ : From lemma 1 we obtain:

$$
A(p)=A^{(N)}(p)=I a_{\nu} p^{\nu}+B, B_{[\alpha \lambda]\left[\alpha^{\prime} \lambda^{\prime}\right]}=B_{\alpha \alpha^{\prime}} \delta_{\lambda \lambda^{\prime}}
$$

and this is recognized as a sum of a generator of translations (the first term) and a generator of an internal symmetry (the second term).

$N=1:$ From lemma 1 we have

$$
A_{\mu}^{(1)}(p)=I a_{\nu \mu} p^{\nu}, a_{\nu \mu}=-a_{\mu \nu}
$$

which implies that

$$
A(p)=A^{(0)}(p)+I a_{\nu \mu} p^{\nu} \frac{1}{i} \frac{\partial}{\partial p_{\mu}} .
$$

The second term is the space part of a generator of $\mathcal{L}$. Subtracting this generator from $A$ one obtains a 0 -order (self adjoint) element of $\mathcal{A}$ which was already shown to satisfy the statement of the proposition.

$N>1: a_{\nu \vec{\mu}}$ is symmetric in $\vec{\mu}$ (by eq. (III.69)). Combining this with eq. (III.72) one obtains:

$$
\begin{aligned}
a_{\nu \mu_{1} \mu_{2} \ldots} & =-a_{\mu_{1} \nu \mu_{2} \ldots}=-a_{\mu_{1} \mu_{2} \nu \ldots}=a_{\mu_{2} \mu_{1} \nu \ldots}= \\
& =a_{\mu_{2} \nu \mu_{1} \ldots}=-a_{\nu \mu_{2} \mu_{1} \ldots}=-a_{\nu \mu_{1} \mu_{2} \ldots}
\end{aligned}
$$

so $a_{\nu \vec{\mu}}=0$ which implies that $A_{\vec{\mu}}^{(N)}=0$ in contradiction to the fact that $A$ is of $\operatorname{order} N$. Thus $N$ is either 0 or 1 .

\section{Comments and Supplements}

In this section we discuss the assumptions of the theorem proved in this work, emphasizing the prospects for relaxing some of them. 


\section{IV.1 Other Signatures and Orbits}

The theorem was proved for signatures of the type $(r, 1)$ (assumption 2) and for representations with momentum support in the forward light cone (assumption 3). All this was needed to assure the compactness of the little group, which implies that its irreducible representation spaces $\left\{\mathcal{H}\left(L^{\alpha}\right)\right\}$ are finite-dimensional. The finiteness of the dimension of the spaces $\{\mathcal{H}(m)\}$ (which are direct sums of $\left\{\mathcal{H}\left(L^{\alpha}\right)\right\}$ ) is essential to all of part 2 of the proof and also plays a key role in the construction of the base of plane-wave states [15]. It was used to construct, using the method of induced representations, a space $\Phi_{\alpha}$ in which the generators of $\mathcal{P}$ are represented. Such a space can be constructed for any representation which can be built by a sequence of inductions, starting with a finite-dimensional representation. Perhaps the proof of the theorem may also be constructed for such types of representations by applying the methods described in chapter 3 successively for each stage of induction.

The infinite dimension of $\left\{\mathcal{H}\left(L^{\alpha}\right)\right\}$ may cause another complication. In this case, the spectrum $\sigma_{\alpha}$ (of the operator $J$ used to represent $\left\{\mathcal{H}\left(L^{\alpha}\right)\right\}$ as a space of functions - see Section A.3) is not necessarily discrete. If it is continuous, $\Omega$ (defined in appendix A.4) is not a countable union of orbits so to consider $\Omega$ as a smooth separable manifold, one must include a differential structure on $\sigma_{\alpha}$; this must be taken into account when checking the smoothness of functions on $\Omega$. If the spectrum is mixed, $\Omega$ is a union of manifolds of different dimension.

Finally, the choice of signature $(r, 1)$ and momenta in the forward light cone has also a physical significance. In this region $p^{0}$ is bounded from below (positive), thus suitable to be interpreted as the energy. In any other case (except for the forward light-like momenta in the case of signature $(r, 1)$ ) the orbits are unbounded in all directions, and therefore the canonical energy is not well defined (Recall that the energy is distinguished from other components of the momentum by being positive and this in an invariant (and therefore well defined) statement only in the case of signature $(r, 1)$.).

The restriction $r \geq 3$ was used twice. In lemma 9 of part 2 of the proof, it assured the existence of a one-parameter subgroup of the little group of a time-like momentum. It also assured that all the projective representations of $\mathcal{P}$ are (equivalent to) true representations (this is true for any signature $(r, s)$ with $r+s \geq 3$ [17]). This was the main motivation in replacing $\mathcal{P}_{0}^{\prime}$ with its covering group $\mathcal{P}$. The problem with projective representations is that they do not lead naturaly to a representation of the generators of the group.

\section{IV.2 The Particle-Type Spectrum}

In part 1 of the proof it was assumed that the mass spectrum is bounded; for part $2, \mathcal{H}(m)$ must be finite-dimensional, which implies that the number of particles with the same mass must be finite; and for part 3 (eq. (III.70) ) the mass spectrum $\mathcal{M}$ must consist of isolated points. Combining all this, one finds that the number of particle types must be finite.

It might be possible to extend part 1 of the proof to an unbounded mass spectrum (as suggested by Coleman and Mandula [1]), so, considering the other restrictions, the 
mass spectrum could be an infinite increasing sequence, diverging to infinity. But some modifications are needed. Recall (section 3.1) that the requirement of a bounded spectrum was used to show the existence of a sufficient variety of physical momenta outside the region $\bar{R} \cup \overline{R^{\dagger}}$ (lemma 3). In the case of an unbounded mass spectrum, $\overline{R_{m}} \cup \overline{R_{m}^{\dagger}}$ is spread over all of $\hat{\mathcal{T}}_{m}$ for $\Delta p_{0}$ in the forward light cone, therefore the required momenta must be looked for between the bands of $\overline{R_{m}} \cup \overline{R_{m}^{\dagger}}$. The width of the bands is determined by $\rho$ (the radius of the support of the function $f$ used to construct $f \cdot A$ ), but it also depends on the angle of intersection of $\hat{\mathcal{T}}_{m}$ and $\hat{\mathcal{T}}_{m_{\alpha}}$ (see figure [11.1), therefore it cannot be bounded uniformly. Roughly speaking, the width increases with the distance from the origin so if the intervals between the masses don't increase accordingly, they will start to overlap far enough from the origin. With these considerations it is possible to show:

\section{Proposition:}

If $\mathcal{M}=\left\{m_{\alpha}\right\}$ is an increasing sequence and $\lim _{\alpha \rightarrow \infty} \frac{m_{\alpha+1}}{m_{\alpha}}=1$ (which means that $\left\{m_{\alpha}\right\}$ increases slower than any geometric series) then, for time-like $\Delta p$ and for $\alpha$ sufficiently large, the (elliptic) bands overlap completely.

This implies that under the assumptions of the proposition, $\hat{\mathcal{T}}_{m} \backslash \bar{R}$ is bounded, therefore for $p_{0}^{\prime}$ large enough, the momenta satisfying lemma 3 don't exist, so the proof for this case can not proceed as described in section 3.

Attempts to deal with this problem can be made in two different approaches:

1. Investigation of the conditions on $\mathcal{M}$ in which the existence of the required momenta can be assured: Using the same methods used to prove the above proposition one may establish conditions on $\mathcal{M}$ for which at least on half of $\hat{\mathcal{T}}_{m}$ the bands occupy arbitrarily small portions of $\hat{\mathcal{T}}_{m}$. This seems sufficient to show the existence of the required momenta, but the proof may be quite complicated technically.

2. Investigation of conditions on $\mathcal{M}$ in which, for a given $\rho>0$ the required momenta exist for $p_{0}^{\prime}$ in some region of $\hat{\mathcal{T}}_{m}$ that approaches all of $\hat{\mathcal{T}}_{m}$ at the limit $\rho \rightarrow 0$. The arguments of part 1 imply now that the support of $f \cdot A$ is outside this region. It remains to show what this says about $A$.

\section{IV.3 The Assumptions on the Scattering Amplitudes}

The regularity of the scattering amplitudes is used only in lemma 3 of part 2 of the proof (see eq. (III.39)), to state that the integrands in the right hand side of

$$
\begin{aligned}
& 2 \operatorname{Im}\left(h^{\dagger}<p^{2}|T| p^{2}>h\right)= \\
& \quad \sum_{m=0}^{\infty} \int_{\hat{\mathcal{T}}_{F}^{m}} d \mu^{m}\left(q^{m}\right)(2 \pi)^{d} \delta^{d}\left(\sum_{1}^{m} q_{j}-\sum_{1}^{2} p_{i}\right) h^{\dagger}<p^{2}|T| q^{m}>^{\dagger}<q^{m}|T| p^{2}>h
\end{aligned}
$$

are non-negative and thus so are the integrals. If an analogous argument for distributions can be given to show that the integrals are non negative (in the sense of generalized 
functions) then it will not be necessary to assume anything about amplitudes between states with more than two particles.

The analyticity of $\left\langle T^{(2,2)}>\right.$ is used many times but most of the time only the analyticity of elastic scattering amplitudes (those connecting states with the same types of particles) is really needed. The only use of the full amplitude is to show the $S^{(2,2)}$-invariance of $\Phi^{(2)}$ and there it is enough to assume that it is smooth. However the distinction between elastic and not elastic amplitudes seems rather artificial, since by performing an internal transformation (mixing particle types) the "new" elastic amplitudes are linear combinations of "old" inelastic amplitudes. Such an argument might be used to show that the analyticity of the elastic amplitudes in fact implies the analyticity (or at least the smoothness) of $\left\langle T^{(2,2)}\right\rangle$. Realizing that the "diagonal" of $\left\langle T^{(2,2)}\right\rangle$ consists only of elastic amplitudes, this seems somehow related to the result that a sesquilinear form is determined by its diagonal, i.e. as a polarization of the form:

$$
(x, y)=\frac{1}{2}[(x+y, x+y)-i(x+i y, x+i y)]+\frac{i-1}{2}[(x, x)+(y, y)] .
$$

\section{IV.4 Super Symmetry}

The proof of the theorem refers actually only to the algebra of generators of symmetry (and not to the symmetry group), therefore most of it can be applied also to supersymmetric generators, as observed in [4]. To include supersymmetry, one has to modify slightly the definition of $\mathcal{A}$ (at the beginning of section 3). In this case $\mathcal{A}$ is a direct sum

$$
\mathcal{A}=\mathcal{A}_{0} \oplus \mathcal{A}_{1} \in L^{\times}\left(\Phi^{(1)} ; \Phi^{(1)^{\prime}}\right)
$$

and the "even" and "odd" elements are distinguished by their action in $\Phi^{(1)} \times \Phi^{(1)}$. For $\varphi_{1}$ either purely even (bosonic) or purely odd (fermionic), and $\varphi_{2}$ arbitrary, (4.2) is replaced by

$$
A \varphi:=(A \otimes I \pm I \otimes A) \varphi=\left(A \varphi_{1}\right) \otimes \varphi_{2} \pm \varphi_{1} \otimes\left(A \varphi_{2}\right),
$$

where the minus sign refers to $A$ and $\varphi_{1}$ both odd and the plus sign to all other combinations (this form can be deduced from the Fock representation of the space of states, where the generators, are bilinears of creation and destruction operators). Comparing to the original definition (in section 3), we observe that $\mathcal{A}_{0}$ is the original $\mathcal{A}$ and therefore the theorem applies fully to $\mathcal{A}_{0}$. As for all of $\mathcal{A}$, among its properties enumerated at the beginning of section 4 , only the last one needs modification:

$4^{\prime}$. If $A \in \mathcal{A}_{i}, B \in \mathcal{A}_{j},(i, j=0$ or 1$), \Phi^{(1)}$ is invariant under $A$ and $B$ and $A B, B A \in L^{\times}\left(\Phi^{(1)} ; \Phi^{(1)^{\prime}}\right)$ then $A B-(-1)^{i j} B A \in \mathcal{A}$.

This property was not used in Part 1, so proposition 1 holds for all of $\mathcal{A}$. In particular, all the generators have the form ([II.69) and commute with the "mass" operator $P^{2}$. Property $\left(4^{\prime}\right)$ entered the proof only in its second part, after Lemma 6, where it lead to the conclusion that $\mathcal{B}^{*}$ is a Lie algebra. This obviously translates here to the statement 
that $\mathcal{B}^{*}$ is a graded Lie algebra. Lemma 6 of part 2 (together with the form of $\mathcal{A}_{0}$, as given by the theorem) was the starting point of [4] and since it continues to hold in general dimension, one can proceed as in [4] to determine the general form of $\mathcal{A}$.

\section{Conclusions}

In this work we investigated the generalization of the Coleman-Mandula theorem to higher dimension. It states that the group of symmetries of the (nontrivial, Poincaréinvariant) scattering matrix $S$ can contain, in addition to the Poincaré symmetries, only Poincaré-invariant symmetries (note that this does not exclude a richer symmetry of the action). The theorem was proved for arbitrarily higher spatial dimension and for a finite number of particle types, all of them massive. Further generalization requires more involved analysis and this was discussed in some detail in the last section.

To put the analysis on a firm basis, with minimal loss in clarity, we used a rigorized version of the Dirac formalism, developed in [14] and applied in [15] to scattering scenarios. Unlike other rigorous formulations of quantum mechanics, in this formalism it is possible to use "a complete set of plane-wave states" to decompose expressions into "vector components" and "matrix elements" in almost the same flexibility as in the original Dirac formalism. This work should also be seen as a demonstration of this flexibility.

\section{Appendix A. Summary of Notation and Concepts}

\section{A.1 The Group $\mathcal{P}(r, s)$}

- $\mathcal{P}_{0} \equiv \mathcal{P}_{0}(r, s)$ is the restricted (identity component of) inhomogeneous pseudoorthogonal group of signature $(r, s)$;

- $\mathcal{L}_{0} \equiv \mathcal{O}_{0}(r, s)$ is the homogeneous part of $\mathcal{P}_{0}(r, s)$;

- $\mathcal{T} \equiv \mathcal{T}_{r+s}$ is the translation group in $\mathbf{R}^{r+s}$;

- $\mathcal{P}$ and $\mathcal{L}$ are the universal covering groups of $\mathcal{P}_{0}$ and $\mathcal{L}_{0}$ respectively.

$\mathcal{P}\left(\mathcal{P}_{0}\right)$ is the semi-direct product of $\mathcal{T}$ and $\mathcal{L}\left(\mathcal{L}_{0}\right)$. The theorem is proved for $s=1$ and $r \geq 3$ (assumption 2) and these are the values assumed also in the Appendices. For these values $\mathcal{L}_{0}=\mathcal{L} / \mathbf{Z}_{2}$. 


\section{A.2 The Momentum Space}

- $\hat{\mathcal{T}}$, "the momentum space": the dual of the translation group $\mathcal{T}$;

- $\hat{\mathcal{T}}_{m}$, "A mass shell": an orbit of $\mathcal{L}$ in $\hat{\mathcal{T}}$; assumed to be in the forward light cone (assumption 3 of the theorem), so the elements $\{p\}$ of $\hat{\mathcal{T}}_{m}$ are characterized by the "mass" $m=\sqrt{p_{\mu} p^{\mu}}$;

- $\mu_{m}$ : the $\mathcal{L}$-invariant non-trivial Radon measure on $\hat{\mathcal{T}}_{m}$ (unique up to a multiplicative constant); it is non-degenerate, in the sense that it does not vanish on open sets;

- $\mathcal{M}$, "the one-particle mass spectrum": the set of masses of the particles of the system; assumption 4 of the theorem implies that it is a finite set;

- $\hat{\mathcal{T}}_{F}:=\bigcup_{m \in \mathcal{M}} \hat{\mathcal{T}}_{m}$ : the physical region in $\hat{\mathcal{T}}$ for one-particle states.

\section{A.3 The Space $\mathcal{H}_{\alpha}$ of $\alpha$-states}

A particle type $\alpha$ is identified with an irreducible representation $U^{\alpha}$ of $\mathcal{P}$ in the space of one-particle states. $\mathcal{H}_{\alpha}$ - "the $\alpha$-states space" is the representation space of $U^{\alpha}$ (the space of all possible states in which there is one particle and it is of type $\alpha$ ).

After a spectral decomposition of $\mathcal{H}_{\alpha}$, one gets:

$$
\mathcal{H}_{\alpha}=\bigoplus_{\lambda \in \sigma_{\alpha}} \mathcal{L}_{\mu_{m_{\alpha}}}^{2}\left(\hat{\mathcal{T}}_{m_{\alpha}}\right)
$$

where

- $\hat{\mathcal{T}}_{m_{\alpha}}$, "the $m_{\alpha}$-mass shell" is the spectrum of the momentum operator $P$ in $\mathcal{H}_{\alpha}$;

- $\sigma_{\alpha}$ is the spectrum of the operator $J \in \mathcal{U}_{\mathcal{P}}$ (where $\mathcal{U}_{\mathcal{P}}$ denotes the universal enveloping algebra of the Lie algebra of $\mathcal{P}$ ) which supplements $P$ to a complete system of commuting observables in $\mathcal{H}_{\alpha}$; assumption 3 of the theorem implies that $\sigma_{\alpha}$ is a finite set;

so, for each $f \in \mathcal{H}_{\alpha}$

$$
\begin{aligned}
{\left[U^{\alpha}\left(P^{\mu}\right) f\right](p, \lambda) } & =p^{\mu} f(p, \lambda) \\
{\left[U^{\alpha}\left(J_{i}\right) f\right](p, \lambda) } & =\lambda_{i} f(p, \lambda)
\end{aligned}
$$

\section{A.4 The One-Particle Space $\mathcal{H}^{(1)}$}

$$
\mathcal{H}^{(1)}=\bigoplus_{\alpha \in I} \mathcal{H}_{\alpha}=\mathcal{L}_{\mu}^{2}(\Omega)
$$

where

- $I$ is the set of particle types (a finite set according to assumption 4 of the theorem); 
- $\Omega:=\left\{(p, \lambda, \alpha) \mid \alpha \in I, \lambda \in \sigma_{\alpha}, p \in \hat{\mathcal{T}}_{m_{\alpha}}\right\}$ (it is an $r$-dimensional separable smooth manifold);

- $\mu$ is the (non-degenerate Radon) measure on $\Omega$ defined by

$$
\int_{\Omega} d \mu(p, \lambda, \alpha) \ldots=\sum_{\alpha \in I} \sum_{\lambda \in \sigma_{\alpha}} \int_{\hat{\mathcal{I}}_{m_{\alpha}}} d \mu_{m_{\alpha}}(p) \ldots
$$

Considering an element of $\mathcal{H}^{(1)}$ as a vector-valued function on the momentum space $\hat{\mathcal{T}}$, we write:

$$
\mathcal{H}^{(1)}=\bigoplus_{m \in \mathcal{M}} \mathcal{L}_{\mu_{m}}^{2}\left(\hat{\mathcal{T}}_{m}, \mathcal{H}(m)\right)
$$

where

- $I(m):=\left\{\alpha \in I \mid m_{\alpha}=m\right\}$ is the set of particle types with mass $m$;

- $\Omega(m):=\left\{[\alpha \lambda] \mid \alpha \in I(m), \lambda \in \sigma_{\alpha}\right\}$ (this is a finite set according to assumptions 3 and 4 of the theorem);

- $N(m)$ is the number of elements in $\Omega(m)$;

- $\mathcal{H}(m)=\mathbf{C}^{N(m)}$ (the $N(m)$-dimensional complex Hilbert space).

When $m=\sqrt{p_{\mu} p^{\mu}}, I(p), \Omega(p), N(p), \mathcal{H}(p)$ stand for $I(m)$ etc. .

With this approach, $\mu$ can be seen as a measure on $\hat{\mathcal{T}}_{F}$ :

$$
\int_{\Omega} d \mu(p, \lambda, \alpha) \ldots=\int_{\hat{\mathcal{T}}_{F}} d \mu(p) \sum_{[\alpha \lambda] \in \Omega(p)} \ldots
$$

where

$$
\int_{\hat{\mathcal{T}}_{F}} d \mu(p) \ldots:=\sum_{m \in \mathcal{M}} \int_{\hat{\mathcal{T}}_{m}} d \mu_{m}(p) \ldots
$$

\section{A.5 The Full Space of States}

The $n$-particle space $\mathcal{H}_{s}^{(n)}$ is a closed subspace of

$$
\mathcal{H}^{(n)}=\overline{\bigotimes_{1}^{n} \mathcal{L}_{\mu}^{2}(\Omega)}=\mathcal{L}_{\mu^{n}}^{2}\left(\Omega^{n}\right)
$$

(the bar denotes closure in $\mathcal{L}_{\mu^{n}}^{2}\left(\Omega^{n}\right)$ ) where

- $\Omega^{n}:=\Omega \times \cdots \times \Omega$ ( $n$ factors $)$

(this is a separable smooth manifold); 
- $\mu^{n}$ is the ( $\mathcal{L}$-invariant non degenerate Radon) measure defined by

$$
\int_{\Omega^{n}} d \mu^{n}\left(p^{n}, \lambda^{n}, \alpha^{n}\right) \ldots:=\int_{\Omega} d \mu\left(p_{1}, \lambda_{1}, \alpha_{1}\right) \ldots \int_{\Omega} d \mu\left(p_{n}, \lambda_{n}, \alpha_{n}\right) \ldots
$$

or, in vector notation:

$$
\int_{\hat{\mathcal{T}}_{F}^{n}} d \mu^{n}\left(p^{n}\right) \ldots:=\int_{\hat{\mathcal{T}}_{F}} d \mu\left(p_{1}\right) \ldots \int_{\hat{\mathcal{T}}_{F}} d \mu\left(p_{n}\right) \ldots
$$

where $\hat{\mathcal{T}}_{F}^{n}:=\hat{\mathcal{T}}_{F} \times \cdots \times \hat{\mathcal{T}}_{F}(n$ factors $)$.

The elements of $\mathcal{H}_{s}^{(n)}$ are those elements of $\mathcal{H}^{(n)}$ which have the right symmetry properties with respect to exchange of identical particles.

The space $\mathcal{H}_{s}$ of all physical states is

$$
\mathcal{H}_{s}=\bigoplus_{n=0}^{\infty} \mathcal{H}_{s}^{(n)}
$$

and thus it is a closed subspace of

$$
\mathcal{H}=\bigoplus_{n=0}^{\infty} \mathcal{H}^{(n)}
$$

For $f \in \mathcal{H}$ we write

$$
f=\sum_{n} f^{n}, f^{n} \in \mathcal{H}^{(n)}
$$

For $f^{n} \in \bigotimes_{1}^{n} \mathcal{H}^{(1)}$ we write

$$
f^{n}=\bigotimes_{i} f_{i}^{n}, f_{i}^{n} \in \mathcal{H}^{(1)}
$$

For $\varphi \in \mathcal{H}^{(n)}, p^{n} \in \Omega^{n}, \varphi\left(p^{n}\right)$ is a vector in

$$
\mathcal{H}\left(p^{n}\right):=\bigotimes_{1}^{n} \mathcal{H}\left(p_{i}\right)
$$

\section{A.6 Gel'fand Triples}

The Gel'fand triples (see section 2.1) for the various spaces defined above are obtained by defining:

$$
\begin{array}{llll}
\text { for } \mathcal{H}_{\alpha} & : & \Phi_{\alpha} & :=\bigoplus_{\lambda \in \sigma_{\alpha}} \mathcal{D}\left(\hat{\mathcal{T}}_{m_{\alpha}}\right) \\
\text { for } \mathcal{H}^{(1)} & : & \Phi^{(1)} & :=\mathcal{D}(\Omega)=\bigoplus_{\alpha \in I} \Phi_{\alpha} \\
\text { for } \mathcal{H}^{(n)} & : & \Phi^{(n)} & :=\mathcal{D}\left(\Omega^{n}\right) \\
\text { for } \mathcal{H}_{s}^{(n)} & : & \Phi_{s}^{(n)} & :=\Phi^{(n)} \cap \mathcal{H}_{s}^{(n)} \\
\text { for } \mathcal{H}^{\prime} & : & \Phi & :=\bigoplus_{n=0}^{\infty} \Phi^{(n)} \\
\text { for } \mathcal{H}_{s} & : & \Phi_{s} & :=\Phi \cap \mathcal{H}_{s}=\bigoplus_{n=0}^{\infty} \Phi_{s}^{(n)}
\end{array}
$$


(The direct sums are as defined for locally convex spaces and are the sets of finite sums of elements.)

The commuting set of observables includes the components of the momentum operator $P^{\mu}$ therefore the basis elements are "plane waves":

$$
\left\{<p^{n}|| n=0,1, \ldots, p^{n} \in \hat{\mathcal{T}}_{F}^{n}\right\}
$$

and the expression that plays the role of the identity operator is

$$
I:=\sum_{n=0}^{\infty} \int_{\hat{\mathcal{T}}_{F}^{n}} d \mu^{n}\left(p^{n}\right)\left|p^{n}><p^{n}\right|
$$

The matrix elements $<q^{m}|A| p^{n}>$ of an operator $A \in L^{\times}\left(\Phi ; \Phi^{\prime}\right)$ are the generalized "values" of the kernels $<A^{(m, n)}>\in \mathcal{D}^{\prime}\left(\Omega^{m} \times \Omega^{n}\right)$ satisfying

$$
(\psi|A| \varphi)=\sum_{m, n=0}^{\infty} \int_{\hat{\mathcal{T}}_{F}^{m}} d \mu^{m}\left(q^{m}\right) \int_{\hat{\mathcal{T}}_{F}^{n}} d \mu^{n}\left(p^{n}\right)\left(\psi\left|q^{m}><q^{m}\right| A\left|p^{n}><p^{n}\right| \varphi\right) .
$$

Since $\varphi\left(p^{n}\right)$ is a vector in $\mathcal{H}\left(p^{n}\right) \equiv \otimes_{1}^{n} \mathcal{H}\left(p_{i}\right)$, eq. (A.20) implies the following interpretation:

$<p^{n} \mid \varphi$ ) (and therefore also $<p^{n} \mid$ ) is a column vector of dimension $\operatorname{dim} \mathcal{H}\left(p^{n}\right)$;

$\left(\psi \mid q^{m}>\right.$ (and therefore also $\left.\mid q^{m}>\right)$ is a row vector;

$<q^{m}|A| p^{n}>$ is a matrix operator from $\mathcal{H}\left(p^{n}\right)$ to $\mathcal{H}\left(q^{m}\right)$.

\section{A.7 The Representation $U$ of $\mathcal{P}$}

\section{A.7.1 The Irreducible Representation $U^{\alpha}$ in $\mathcal{H}_{\alpha}$}

We denote

$$
p_{m}:=(m, 0, \ldots, 0) \text { ("the rest frame") }
$$

and choose a smooth function $p \mapsto \Lambda_{p}$ from $\hat{\mathcal{T}}_{m}$ to $\mathcal{L}$ obeying:

1. $\Lambda_{p_{m}}=1$ (the unit matrix)

2. $\Lambda_{p} p_{m}=p, \forall p \in \hat{\mathcal{T}}_{m}$

Now $U^{\alpha}$ is (for all $(\Lambda, a) \in \mathcal{P}, f \in \mathcal{H}_{\alpha}, p \in \hat{\mathcal{T}}_{m_{\alpha}}$ )

$$
\left[U^{\alpha}(\Lambda, a) f\right](p)=e^{i p \cdot a} L^{\alpha}(\Delta(\Lambda, p)) f\left(\Lambda^{-1} p\right)
$$

where $\Delta(\Lambda, p)$ is defined by

$$
\Delta(\Lambda, p)=\Lambda_{p}^{-1} \Lambda \Lambda_{\Lambda^{-1} p} \in \mathcal{L}\left(p_{m_{\alpha}}\right)
$$

and $L^{\alpha}$ is a continuous unitary irreducible matrix representation of the little group $\mathcal{L}\left(p_{m_{\alpha}}\right)$, which in this case is the universal covering group of $\mathcal{O}(r)$. 


\section{A.7.2 The Representation in $\mathcal{H}$}

The representation in $\mathcal{H}^{(1)}$ is $U^{(1)}=\bigoplus_{\alpha \in I} U^{\alpha}$ so (for all $(\Lambda, a) \in \mathcal{P}, f \in \mathcal{H}^{(1)}, p \in \hat{\mathcal{T}}_{F}$ )

$$
\left[U^{(1)}(\Lambda, a) f\right](p)=e^{i p \cdot a} L^{(p)}(\Delta(\Lambda, p)) f\left(\Lambda^{-1} p\right)
$$

where $L^{(p)}=\bigoplus_{\alpha \in I(p)} L^{\alpha}$ is the (reducible) unitary representation of $\mathcal{L}\left(p_{m}\right)$ in $\mathcal{H}(m)$ $\left(m=\sqrt{p_{\mu} p^{\mu}}\right)$.

The representation $U^{(n)}$ in $\otimes_{1}^{n} \mathcal{H}^{(1)}$ is

$$
U^{(n)}\left(f_{1} \otimes \cdots \otimes f_{n}\right)=\left(U^{(1)} f_{1}\right) \otimes \cdots \otimes\left(U^{(1)} f_{n}\right), \forall f_{i} \in \mathcal{H}^{(1)} .
$$

This is extended to $\mathcal{H}^{(n)}$ by continuity and to $\mathcal{H}$ by linearity.

\section{A.7.3 The Matrix Elements of $U^{(1)}(\mathcal{P})$}

The action of $U^{(1)}$ on the base vectors is

$$
U^{(1)}(\Lambda, a)|p>=| \Lambda p>e^{i a \cdot \Lambda p} L^{(p)}(\Delta(\Lambda, \Lambda p))
$$

and with components:

$$
U^{(1)}(\Lambda, a)\left|p, \lambda, \alpha>=e^{i a \cdot \Lambda p} L^{\alpha}(\Delta(\Lambda, \Lambda p))_{\lambda^{\prime} \lambda}\right| \Lambda p, \lambda^{\prime}, \alpha>.
$$

The matrix elements of $U^{(1)}$ are

$$
<p^{\prime}\left|U^{(1)}(\Lambda, a)\right| p>=e^{i a \cdot \Lambda p} L^{(p)}(\Delta(\Lambda, \Lambda p)) \delta_{\mu}\left(p^{\prime}-\Lambda p\right)
$$

and with components:

$$
<p^{\prime}, \lambda^{\prime}, \alpha^{\prime}\left|U^{(1)}(\Lambda, a)\right| p, \lambda, \alpha>=e^{i a \cdot \Lambda p} \delta_{\alpha^{\prime} \alpha} L^{\alpha}(\Delta(\Lambda, \Lambda p))_{\lambda^{\prime} \lambda} \delta_{\mu}\left(p^{\prime}-\Lambda p\right) .
$$

\section{A.8 Generators of Symmetry}

A one-parameter symmetry group $g(t)$ of $S$ is represented in $\mathcal{H}^{(n)}$ by a unitary representation $U^{(n)}$. The generator $A_{g}^{(n)}$ of $U^{(n)}(g(t))$ is defined by

$$
\left(\psi\left|A_{g}^{(n)} \varphi>:=\frac{1}{i} \frac{d}{d t}\left(\psi, U^{(n)}(g(t)) \varphi\right)\right|_{t=0} \quad, \quad \forall \varphi, \psi \in \Phi^{(n)} .\right.
$$

$A_{g} \equiv A_{g}^{1}$ is assumed (assumption 5 of the theorem) to be an element of $L^{\times}\left(\Phi^{(1)} ; \Phi^{(1)^{\prime}}\right.$ ) (a continuous antilinear map from $\Phi^{(1)}$ to $\left.\Phi^{(1)^{\prime}}\right)$ and it is self adjoint. If $g(t)$ is a subgroup of $\mathcal{P}, A_{g}$ is, by construction of $\Phi^{(1)}$, a continuous operator in $\Phi^{(1)}$ (and therefore certainly satisfies assumption 5).

Between elements of $\bigoplus_{1}^{n} \Phi^{(1)}$ :

$$
A_{g}^{(n)}=\left(A_{g} \otimes I \otimes \cdots \otimes I\right)+\left(I \otimes A_{g} \otimes \cdots \otimes I\right)+\cdots+\left(I \otimes I \otimes \cdots \otimes A_{g}\right)
$$


Being a generator of symmetry, $A_{g}$ satisfies (when $S^{*} \psi, S \varphi \in \Phi$ )

$$
\left(S^{*} \psi\left|A_{g} \varphi>=<A_{g} \psi\right| S \varphi\right)
$$

In particular, if $S$ and $A_{g}$ are operators in $\Phi$ then

$$
\left[A_{g}, S\right]=0 \text { in } \Phi .
$$

Also, if $A_{g}$ is a continuous operator in $\Phi$ (e.g. a generator of $\mathcal{P}$ ) then (A.33) holds, with the commutators defined to be

$$
\left[A_{g}, S\right]=A_{g}^{\prime} S-S A_{g}
$$

where $A_{g}^{\prime}$ is the dual of $A_{g}$ and $S$ is considered as an operator from $\Phi$ to $\Phi^{\prime}$.

\section{A.9 Scattering Amplitudes}

The S-matrix $S$, being unitary, can be identified as an element of $L^{\times}\left(\Phi ; \Phi^{\prime}\right)$. As such, it has a corresponding kernel $\langle S\rangle$ (more precisely - kernels $<S^{(m, n)}>\in \mathcal{D}\left(\Omega^{m} \times \Omega^{n}\right)$ ).

The translation invariance of $S$ implies that $<S>$ has the following form

$$
<S^{(m, n)}>=<I^{(m, n)}>-i(2 \pi)^{d} \delta^{d}\left(\sum_{1}^{m} q_{j}-\sum_{1}^{n} p_{i}\right)<T^{(m, n)}>
$$

where $d=r+1$ is the dimension of the momentum space, $-i(2 \pi)^{d}$ is a conventional normalization factor and $\langle T\rangle$ is a generalized function on the submanifold of $\Omega^{m} \times \Omega^{n}$ defined by the constraint

$$
\sum_{1}^{m} q_{j}-\sum_{1}^{n} p_{i}=0
$$

(this is the precise formulation of energy-momentum conservation). The values of $<T>$ are called "scattering amplitudes".

Since $S$ is $\mathcal{L}$-invariant, $\langle T>$ depends only on $\mathcal{L}$-invariant quantities. In particular, its dependence on the momenta is only through $\mathcal{L}$-invariant functions of the momenta.

The unitarity of $S$ leads to

\section{The Optical Theorem:}

$$
\begin{aligned}
& <p^{n}|T| p^{n}>-<p^{n}|T| p^{n}>^{\dagger}= \\
& \quad i \sum_{m=0}^{\infty} \int_{\hat{\mathcal{T}}_{F}^{m}} d \mu^{m}\left(q^{m}\right)(2 \pi)^{d} \delta^{d}\left(\sum_{1}^{m} q_{j}-\sum_{1}^{n} p_{i}\right)<p^{n}|T| q^{m}>^{\dagger}<q^{m}|T| p^{n}>
\end{aligned}
$$

where "†" denotes the Hermitian conjugation of (matrix) maps between the spaces $\mathcal{H}\left(p^{n}\right)$.

\section{Appendix B. Two-Particle Scattering}




\section{B.1 The $\mathcal{L}$-Invariant Variables}

The scattering amplitudes depend on the momenta through $\mathcal{L}$-invariant functions. We show here that for the scattering between two-particle states

$$
\left(p_{1}, p_{2}\right) \longrightarrow\left(p_{1}^{\prime}, p_{2}^{\prime}\right)
$$

(where the momenta are, according to assumption 3 of the theorem, in the forward light cone) these functions can be chosen to be the traditional

$$
s:=\left(p_{1}+p_{2}\right)^{2}
$$

and

$$
t:=\left(p_{1}^{\prime}-p_{1}\right)^{2}
$$

Explicitly we show that $s$ and $t$ determine the momenta in the process ( $\overline{B .1})$ up to an $\mathcal{L}$-transformation (represented by an $\mathcal{L}_{0}$ transformation).

We start with the identity

$$
\left(p_{1}+p_{2}\right)^{2}=m_{1}^{2}+m_{2}^{2}+2 E_{1} E_{2}-2 \overrightarrow{p_{1}} \cdot \vec{p}_{2} .
$$

Since $\vec{p}_{1} \cdot \vec{p}_{2} \leq\left|\vec{p}_{1}\right|\left|\vec{p}_{2}\right|<E_{1} E_{2}$, we have $\left(p_{1}+p_{2}\right)^{2}>0$, which means that $p_{1}+p_{2}$ is time-like and can be transformed, by an $\mathcal{L}_{0}$-transformation to the "rest frame", where $\vec{p}_{1}+\vec{p}_{2}=0$. Then one can perform a rotation (an element of $\mathcal{O}_{0}(r)$, which is an $\mathcal{L}_{0}$-transformation), to align $\vec{p}_{1}$ along the $e_{1}$ axis, obtaining $\vec{p}_{1}=(p, 0, \ldots, 0)$. The equality $\vec{p}_{1}+\vec{p}_{2}=0$ is not affected by this transformation, so $\vec{p}_{2}=(-p, 0, \ldots, 0)$ and the conclusion is that the initial state is characterized by one variable $p$, that can be expressed by the invariant variable

$$
s=\left(p_{1}+p_{2}\right)^{2}=m_{1}^{2}+m_{2}^{2}+2 \sqrt{\left(m_{1}^{2}+p^{2}\right)\left(m_{2}^{2}+p^{2}\right)}-2 p^{2} .
$$

(if $m_{1}=m_{2}=m$, this simplifies to $s=4\left(m^{2}+p^{2}\right)$.)

Momentum conservation gives

$$
\vec{p}_{1}^{\prime}+\vec{p}_{2}^{\prime}=\vec{p}_{1}+\vec{p}_{2}=0
$$

Substituting $\left(p^{\prime}\right)^{2}=\left(\vec{p}_{1}{ }^{\prime}\right)^{2}=\left(\vec{p}_{2}{ }^{\prime}\right)^{2}$ in the energy conservation equation, one obtains:

$$
\sqrt{\left(\left(m_{1}^{\prime}\right)^{2}+\left(p^{\prime}\right)^{2}\right)}+\sqrt{\left(\left(m_{2}^{\prime}\right)^{2}+\left(p^{\prime}\right)^{2}\right)}=\sqrt{\left(m_{1}^{2}+p^{2}\right)}+\sqrt{\left(m_{2}^{2}+p^{2}\right)}
$$

and this has at most one solution for $\left(p^{\prime}\right)^{2}$. (Such solution exists iff $\sqrt{s} \geq m_{1}^{\prime}=m_{2}^{\prime}$; for an elastic scattering $\left(m_{i}^{\prime}=m_{i}\right)$ this is $p^{\prime}=p$.)

It is left to determine the direction of $\vec{p}_{1}{ }^{\prime}$. Rotating around $\vec{p}_{1}$, one can bring $\vec{p}_{1}{ }^{\prime}$ to the $\left(e_{1} e_{2}\right)$ plane (notice that such rotation doesn't affect $\vec{p}_{1}, \vec{p}_{2}$ and $p_{1}+p_{2}$ ). So, to characterize the final state, it is enough to give the angle between $\vec{p}_{1}$ and $\vec{p}_{1}{ }^{\prime}$, and this can be expressed by the invariant variable $t=\left(p_{1}-p_{1}^{\prime}\right)^{2}$. 


\section{B.2 The $S^{(2,2)}$-Invariance of $\Phi^{(2)}$}

In this subsection we show that $\Phi^{(2)}$ is $S^{(2,2)}$-invariant and comment about the $S$-invariance of $\Phi$.

First consider the support of $(S \varphi)^{m}$ for an arbitrary $\varphi \in \Phi$. Since $S$ conserves energy and momentum, $\left(p_{1}^{\prime}, \ldots, p_{m}^{\prime}\right)$ can be in the momentum support of $(S \varphi)^{m}$ only if there exists an integer $n$ and $\left(p_{1}, \ldots, p_{n}\right)$ in the momentum support of $\varphi^{n}$ that satisfies $\sum_{1}^{m} p_{j}^{\prime}=\sum_{1}^{n} p_{i}$. For each $n$, the momentum support $\left\{\left(p_{1}, \ldots, p_{n}\right) \mid \varphi^{n}\left(p_{1}, \ldots, p_{n}\right) \neq 0\right\}$ of $\varphi$ is bounded in $\hat{\mathcal{T}}_{F}^{n}$ (where $\hat{\mathcal{T}}_{F}$ is the "physical region" in $\hat{\mathcal{T}}$, see section A.2) and thus $\left\{\sum_{1}^{n} p_{i} \mid \varphi^{n}\left(p_{1}, \ldots p_{n}\right) \neq 0\right\}$ is bounded in the momentum space $\hat{\mathcal{T}}$. $\varphi$ has a finite number of non-vanishing components $\left\{\varphi^{n}\right\}$, thus $\left\{\sum_{1}^{n} p_{i} \mid n=0,1, \ldots, \varphi^{n}\left(p^{n}\right) \neq 0\right\}$ is also bounded (being a finite union of bounded sets). So we conclude that for $\left(p_{1}^{\prime}, \ldots, p_{n}^{\prime}\right)$ in the momentum support of $(S \varphi)^{m}, \sum_{1}^{m} p_{j}^{\prime}$ is restricted to a bounded set in $\hat{\mathcal{T}}$, and in particular, $\sum_{1}^{m} E_{j}^{\prime}$ is bounded. But $\left\{p_{j}^{\prime}\right\}$ are in the forward light cone, so $E_{j}^{\prime}>0, \forall j$, and thus each $E_{j}^{\prime}$ is bounded separately. $\vec{p}_{j}^{\prime}$ is bounded by $E_{j}^{\prime}$ so $p_{j}$ is bounded in $\hat{\mathcal{T}}$. Therefore the momentum support of $(S \varphi)^{m}$ is bounded in $\hat{\mathcal{T}}_{F}^{m}$ and thus compact; the finite dimension of $\mathcal{H}\left(p_{1}^{\prime}, \ldots, p_{m}^{\prime}\right)$ then implies that $(S \varphi)^{m}$ has compact support in $\Omega^{m}$.

The smoothness of $S^{(2,2)} \varphi \equiv(S \varphi)^{2}$, for $\varphi \in \Phi^{(2)}$ follows from the analyticity of the scattering amplitudes $\left\langle T^{(2,2)}\right\rangle$, so we can conclude that $S^{(2,2)} \varphi$ is in $\Phi^{(2)}$.

If, in addition, all the scattering amplitudes are smooth, one can show that $\Phi$ is $S$ invariant. For $\varphi \in \Phi$, the smoothness of the scattering amplitudes implies that $(S \varphi)^{m}$ is smooth and the above analysis shows that $\operatorname{supp}(S \varphi)^{m}$ is compact, which means that $(S \varphi)^{m} \in \Phi^{(m)}$. It is left to show that $(S \varphi)^{m}=0$ for $m$ large enough. This follows from

$$
\sum_{1}^{n} E_{i}=\sum_{1}^{m} E_{j}^{\prime} \geq \sum_{1}^{m} m_{\alpha_{j}} \geq m \inf (\mathcal{M})
$$

since $\operatorname{Inf}(\mathcal{M})$ is positive (according to assumption 3 and 4 of the theorem), so for $m$ large enough, (B.8) cannot be satisfied.

\section{References}

[1] S. Coleman and J. Mandula, Phys. Rev. 159 (1967) 1251

[2] D. V. Volkov and V. P. Akulov, Phys. Lett. B46 (1973) 109.

[3] J. Wess and B. Zumino, Nuc. Phys. B70 (1974) 39.

[4] R. Haag, J. T. Łopuszański, and M. Sohnius, Nuc. Phys. B88 (1975) 257.

[5] L. P. Horwitz, and C. Piron, Helv. Phys. Acta 46 (1973) 316;

L. P. Horwitz, R. I. Arshansky and A. C. Elitzur, Found. of Phys. 18 (1988) 1159;

L. P. Horwitz, Found. of Phys. bf 22 (1992) 421. 
[6] I. T. Todorov, Phys. Rev. D3 2351 (1971).

H. W. Crater and P. Van Alstine, Nuc. Phys. B (Proc. Suppl.) 6 (1989) 271-274.

J. Bijtebier, Nuc. Phys. B (Proc. Suppl.) 6 (1989) 278-280.

[7] This by itself is not yet a contradiction to the theorem since this extended symmetry is a symmetry of the equations of motion and not necessarily of the $S$ matrix.

[8] For example, from the structure of the GLA in a system without scalar generators, as determined by the theorem of Haag et al. one gets that in the rest frame of the system $\left\{Q_{i}, Q_{j}\right\}=\delta_{i j} H$ and $\left[Q_{i}, H\right]=0$ where $H$ is the hamiltonian and $Q_{i}$ are the odd generators. this is the starting point of the Witten model (E. Witten, Nuc. Phys. B185 (1981) 513) which is a simple model in supersymmetric quantum mechanics. It would be desirable to have an analogue of such a model in relativistic quantum mechanics, as described in the introduction. This could be important in the development of a theory for a particle with spin.

[9] J. M. Amigó and H. Reeh, Fortschr. Phys. 36 (1988) 929; and references quoted there.

[10] P. Buchholz, J. T. Łopuszański, and Sz. Rabsztyn, Nucl. Phys. B263 (1986) 155.

[11] W. D. Garber, J. Math. Phys. 24 (1983) 1256.

[12] D. Strube, J. Phys. A18 (1985) 2603; J. Math. phys 31 (1990) 2244; 33 (1992) 808.

[13] P. A. M. Dirac, The Principles of quantum Mechanics, Clarendon Press, Oxford, England, 1930 (1st eddition), 1947 (3rd eddition).

[14] J. E. Roberts, J. Math. Phys. 7 (1966) 1097; Commun. Math. Phys. 3 (1966) 98.

A. Böhm, The Rigged Hilbert Space in Quantum Physics, in Boulder Lectures in Theoretical Physics, A.O.Barut (ed.), vol. 9A (1966)

J.-P. Antoine, J. Math. Phys. 10 (1969) 53, 2276.

[15] L.P. Horwitz and O. Pelc, Construction of a Complete Set of States in Relativistic Scattering Theory, RI-1-96, TAUP 2312-95 preprint.

[16] I. M. Gel'fand, G. E. Shilov, and N. Ya. Vilenkin, Obobshchennye Funktsii $i$ Deistviya Nad Nimi, Gosudarstvennoe Izdatel'stvo Fiziko-Matematicheskoi Literatury, Moskow, 1958-1960 Vols. I-V. English translation: Generalized Functions, Academic Press Inc., New York, 1964.

[17] V. Bargmann, Ann. Math. 59 (1954) 1. 
Captions

Figure III.1: The physical region $\hat{\mathcal{T}}_{F}$

Figure III.2: A cross section of the momentum space at the plane $\left(p_{x}, E\right)$

Figure [II.3: A projection of $\hat{\mathcal{T}}_{m}$ on the $\vec{p}$-hyperplane 